\title{
EXPONENTIAL STABILITY OF DISTRIBUTED PARAMETER SYSTEMS GOVERNED BY SYMMETRIC HYPERBOLIC PARTIAL DIFFERENTIAL EQUATIONS USING LYAPUNOV'S SECOND METHOD*
}

\author{
Abdoua Thhousso ${ }^{1,2}$, Thibaut Besson ${ }^{1}$ And Cheng-Zhong Xu ${ }^{1}$
}

\begin{abstract}
In this paper we study asymptotic behaviour of distributed parameter systems governed by partial differential equations (abbreviated to PDE). We first review some recently developed results on the stability analysis of PDE systems by Lyapunov's second method. On constructing Lyapunov functionals we prove next an asymptotic exponential stability result for a class of symmetric hyperbolic PDE systems. Then we apply the result to establish exponential stability of various chemical engineering processes and, in particular, exponential stability of heat exchangers. Through concrete examples we show how Lyapunov's second method may be extended to stability analysis of nonlinear hyperbolic PDE. Meanwhile we explain how the method is adapted to the framework of Banach spaces $L^{p}, 1<p \leq \infty$.
\end{abstract}

Mathematics Subject Classification. 37L15, 37L45, 93C20.

Received November 2, 2005. Revised September 4, 2006 and December 7, 2007.

Published online May 30, 2008.

\section{INTRODUCTION}

Lyapunov's second method, called also Lyapunov's direct method, is one of efficient and classical methods for studying asymptotic behavior and stability of the dynamical systems governed by ordinary differential equations. It dates back to Lyapunov's thesis published at the beginning of the twentieth century [22]. The applications of the method and its theoretical development have been marked by the works of LaSalle and Lefschetz in the past $[19,35]$ and numerous successful achievements later in the control of mechanical, robotic and aerospace systems $(c f . \quad[3,11,15,24,25,38])$. Since three decades the Lyapunov direct method has found extensions in the studies of feedback stabilization and feedback stabilizability of distributed parameter systems governed by partial differential equations (PDE) (see $[1-4,8,10,13,17,18,32]$ ). Up to today numerous interesting results have been obtained using the elaborated techniques adapted to the classical PDEs such as the wave equation, the beam equation and the Petrovsky system. On the same time we have seen appear a class of novel distributed parameter systems from the chemical engineering and described by nonlinear PDE which are

\footnotetext{
Keywords and phrases. Hyperbolic symmetric systems, partial differential equations, exponential stability, strongly continuous semigroups, Lyapunov functionals, heat exchangers.

* Each correspondence should be sent to the last author.

${ }^{1}$ LAGEP, Bâtiment CPE, Université Claude Bernard, Lyon I, 43 Boulevard du 11 Novembre 1918, 69622 Villeurbanne, France.

2 Departement de Mathématiques et Informatique, Université Abdou Moumouni de Niamey, BP 10662, Niger;

xu@lagep.univ-lyon1.fr
} 
different from the classical PDE (cf. [23,29]). To obtain final products of high quality it becomes necessary to supervise and control the evolution of the corresponding dynamical processes based on more sophisticated models, established by the population balance or the conservation law (see $[14,28]$ ). The typical examples of these chemical engineering processes are crystallization processes and polymerization processes. They are all described by hyperbolic PDEs and nonlinear ordinary differential equations which are coupled via boundary conditions or/and through the dynamical equations. However the dynamical behavior of these models is not as well studied as that of the classical PDE in mathematical physics. The objective of our paper is to investigate these mathematical models in terms of stability using Lyapunov's direct approach.

Our studies begin by linear symmetric hyperbolic systems with two independent variables: time and space. The achieved results and the developed method are generalizable to nonlinear hyperbolic PDEs with more spatial variables. Precisely we elaborate suitable Lyapunov functionals to prove exponential stability of distributed parameter systems governed by hyperbolic PDEs for the demand from chemical engineering. As the same as in the case of finite dimensional dynamical systems, the constructed Lyapunov functionals are not only useful and efficient in the proof of stability but also deserve being considered for doing its control design or stabilization later $(c f$. $[9,34])$. Adapted Lyapunov functionals are though simple to understand but difficult to find, since we do not have at our disposal a systematic way to its construction. The reader will realize how it is not immediate to prove an energy like functional to be a Lyapunov functional in the case of classical mathematical physics equations $[1,2,17,18]$. Motivated by automatic control of high-performance chemical engineering processes, our objective is to extend the Lyapunov direct method to deal with stability of more symmetric hyperbolic PDE systems and to fill in the lack of results in this domain.

Prior to introduction of our results we present a review on some remarkable progresses recently gained in this direction. Our concern is not to provide a survey on the studies of stability but to group together the materials somehow near and understandable to our present and future investigations. By this review we try to indicate how our results should be improved along these progresses.

In [18] the authors have applied with success Lyapunov's direct method to investigate the boundary stabilizability of the wave equation. Actually they have considered the wave equation $y_{t t}-\Delta y=0$ on a bounded domain $\Omega$ with smooth boundary $\partial \Omega$, subject to mixed boundary condition: the Dirichlet $y=0$ on $\Gamma_{1}$ and the Neumann $\frac{\partial y}{\partial n}=u$ on $\Gamma_{0}$, where $\left(\Gamma_{0}, \Gamma_{1}\right)$ is a partition of $\partial \Omega$ such that each of them has non empty interior. Is the dissipative boundary feedback law $u=-b(x) y_{t}$, with $b(x) \geq 0$ in $\Gamma_{0}$, sufficient to guarantee a global asymptotic stability: every solution (corresponding to initial data of finite energy) decays exponentially to zero in the energy space as $t \rightarrow \infty$ ? They have proved that exponential stabilization of the system is achieved if the spatial dimension is not greater than 3 and if the partition $\left(\Gamma_{0}, \Gamma_{1}\right)$ is suitably chosen. The result is notably general in the sense that there is no constraint on the domain $\Omega$. In other words all the wave vibration is forced to disappear by the velocity feedback control properly designed on the boundary. The mathematical proof has been done by using the total energy as Lyapunov functional candidate. To prove exponential stability of the closed-loop system it has been necessary to construct another functional which is equivalent to the energy functional. By the method of multipliers, Grisvard's inequality and a compactness-uniqueness argument they have derived a differential inequality for these functionals which has led to precise estimates on the exponential decay of the solutions. The approach has been generalized to the semilinear wave equation $y_{t t}-\Delta y+f(y)=0$ with some nonlinear function $f$. The approach that we adopt in the present paper is similar to their approach. However we do not need the method of multipliers because necessary estimates can be obtained by direct computations in our cases. Though the shallow water system that we consider is a variant of the wave equation, we can not deduce its exponential stability from the result of [18] because of the boundary conditions which are different. Additionally, for the linear wave equation exponential stabilizability implies exact controllability by a principle from [32]. This principle is also valid for our symmetric hyperbolic systems. However exact controllability is true in a more general context [7].

Indirect boundary stabilization of weakly coupled wave equations has been investigated in [1] using Lyapunov's direct method. The vibrating or conservative system under study is constituted of two subsystems governed by the same wave equation and coupled via a weak symmetric zero order operator. Each wave equation is 
equipped with the Dirichlet boundary condition. One of them is stabilized by means of a well-known boundary stabilizing feedback law (see [17], p. 104, or [18]). The other is free of control. What is the effect of such a partial stabilization on the decay of solutions for the coupled systems as the time goes to infinity? Is the partial stabilization sufficient to stabilize the coupled systems? Mathematically exponential stability is not available for the whole system with such a configuration of stabilization and weak coupling [2]. Nevertheless asymptotic stability of the whole system has been proved by using the energy as Lyapunov functional [1] in the case where the coupling is sufficiently weak. The proof has made use of some integral inequalities on the Lyapunov functional. The decay rate of the energy has been estimated to be polynomial in function of time. The method has been extendible to other vibrating systems with different weak couplings (see $[1,2])$. Similarly indirect internal stabilization of weakly coupled evolution equations has been studied in a more general context [2]. The authors have raised up the same question of stabilizability by the partially stabilizing feedback law which consists to apply viscous damping force (proportional to the velocity) on one of the evolution equations. They have got the same answer as in the case of the indirect boundary stabilization. In particular the stabilization result has been established for the wave equation on a non empty star-shaped bounded open set in $\mathbb{R}^{N}$ and without any specific constraint on the spatial dimension $N \geq 1$. In these works $[1,2]$ the considered systems are linear, the energy plays the role of Lyapunov functionals and the method of multipliers is applied to prove the energy inequalities. The asymptotic decay rate of solutions has been estimated in terms of Hilbert norms. However no further indication has been given to what is concerned with nonlinearities and Banach norms. Although the systems for $N=1$ in $[1,2]$ can be transformed into dissipative symmetric hyperbolic systems that we consider in the present paper, they do not verify our hypothesis H1-H3 in the next section. These systems are worthy of our further investigations.

The paper [12] deals with asymptotic stability of a damped string equation (or 1D wave equation): $w_{t t}+$ $2 a(x) w_{t}=w_{x x}$ where the internal damping $a(x)$ can change sign. Physically speaking viscous damping force of indefinite sign is applied on the string fixed at the extreme ends. If the damping term $a(x)$ is nonnegative and positive on some open subset, then the damped system is exponentially stable. When the damping term is of indefinite sign and sufficiently large, the system exhibits unstable solutions. Some sufficient condition has been proposed in [12] to guarantee exponential stability of the damped system in the case that the damping (i.e., $\left.\|a\|_{L^{\infty}}\right)$ is sufficiently small. For example, the damped system is exponentially stable if the projection component of $a(x)$ is greater than a fixed positive number along the square function of every D'Alembertian eigenfunction. Each projection component represents the real part of its corresponding eigenvalue to the damped system. In the considered case the largest real part of the eigenvalues of the damped system determines the growth rate of its semigroup and so leads to its exponential stability (see also [30]). The result has given some insight into the indefinite damping phenomenon. However the approach has relied essentially on a method of spectral analysis and perturbations, and as a consequence, seems difficult to be extended for vibrating systems of higher spatial dimension. Actually, via a D'Alembert transformation the damped system can be written as symmetric hyperbolic PDE, with the damping term only on the internal domain. However the system does not belong to the class of systems under our consideration, where essentially the boundary dissipation is put forward.

The paper [4] has studied the asymptotic behaviour of global smooth solutions for general entropy dissipative hyperbolic systems of balance law. The systems are supposed to satisfy the strictly entropy dissipative condition and the Shizuta-Kawashima condition. It has been proved in [4] that global smooth solutions approach constant equilibrium state in the $L^{p}$ norm at a rate $O\left(t^{-\frac{m}{2}\left(1-\frac{1}{p}\right)}\right)$, with $p \geq 2$ and $m \geq 1$, as $t \rightarrow \infty$. The result has been established from the linearized system around a constant equilibrium state, for which the authors have been able to estimate the large time behaviour of the corresponding Green kernel. The linearized system is written in the conservative-dissipative form. The Shizuta-Kawashima condition implies that the dissipative term, appearing only in the dissipative part, has effective influences on every component of the solution, i.e., as well on the dissipative part as on the conservative part. The result is valid for entropy dissipative hyperbolic PDEs with constant coefficients, with unbounded spatial domain $\mathbb{R}^{m}, m \geq 1$, and without boundary condition. The symmetric hyperbolic PDEs that we study has for spatial domain a bounded open set in $\mathbb{R}^{m}$ and they are 
equipped with boundary conditions. The energy dissipation is effective on the boundary. Via the boundary coupling all the solutions decay exponentially to zero in the $L^{2}$ norm as $t \rightarrow \infty$, while by applying the result of [4] smooth solutions converge to zero with a polynomial decay rate. Different boundary conditions give rise to different asymptotic behaviours of solutions. The boundary dissipation in our cases has the similar effect to that of the strictly entropy dissipative part in [4] concerning asymptotic behaviour of solutions. However we investigate not only the asymptotic behaviour of solutions but also their stability in the sense of dynamical systems.

After this short review on the recent results of stability analysis by Lyapunov's direct method we organize the rest of the paper as follows. The next section is devoted to describe the structure of the symmetric hyperbolic systems that we will study, and to present the essential result that we will prove with Lyapunov functionals. In Section 3 we construct Lyapunov functionals to prove the essential theorem. Section 4 presents some applications of the essential result and, through concrete examples, different generalizations to nonlinear cases and higher spatial dimension. Section 5 shows how the Lyapunov method is applied to stability analysis in the framework of general Banach spaces, specifically in the space of continuous functions with the uniform topology. The last section contains our conclusions and perspectives.

\section{Statement of the MAin Result}

In [31] Rauch and Taylor have studied the exponential decay of solutions of hyperbolic partial differential equations in bounded domains. In particular they have proved that a dynamical system described by dissipative symmetric hyperbolic PDE with two independent variables is exponentially stable if there is some real dissipation of the energy on the boundary. The proof has been established using the method of characteristics [31]. This class of systems have been considered by [32] in studying the problem of exact controllability. There the exponential stability of these systems has been obtained from the exact controllability result. In both [31,32] the proofs are long and indirect in the sense that some observability inequality needs to be proved. Here we propose a new proof of the stability result for this class of systems using a Lyapunov functional. Our proof is direct and simple. It is an advantage of the Lyapunov direct method. Another advantage of the Lyapunov direct method is that it is not limited to the domain of single space variable as reviewed in the Introduction. Moreover the Lyapunov direct method is adapted to deal with nonlinearities (cf. [32]). The reader is referred to $[8,13,15,26,40]$ for other applications of the Lyapunov approach.

In our previous paper [40], using a Lyapunov functional we have proved the stability result of Rauch and Taylor by supposing that some matrix $b(x)$ in the system is symmetric. Here we propose another Lyapunov functional for the proof without any restriction on $b(x)$. Hence the proof in this paper is a generalization of the previous one.

The symmetric hyperbolic system studied in this paper is governed by the following PDE:

$$
\left\{\begin{array}{l}
\frac{\partial R(x, t)}{\partial t}=a(x) \frac{\partial R(x, t)}{\partial x}+b(x) R(x, t) \\
R^{-}(0, t)=D_{0} R^{+}(0, t) \\
R^{+}(1, t)=D_{1} R^{-}(1, t) \\
R(x, 0)=R_{0}(x)
\end{array}\right.
$$

where $(x, t) \in(0,1) \times \mathbb{R}^{+}$. The evolution of the system (2.1) is determined by its boundary condition and its initial condition $R_{0}$. Note that $R(x, t)$ is a vector function of $x$ and $t$ that we partition as

$$
R(x, t)=\left(\begin{array}{c}
R^{-}(x, t) \\
R^{+}(x, t)
\end{array}\right)
$$

such that $R^{-}(x, t) \in \mathbb{R}^{p}, R^{+}(x, t) \in \mathbb{R}^{q}$ and $p+q=n$. The matrix functions $a(x)$ and $b(x)$ have their values in $\mathbb{R}^{n \times n}$. The matrices $D_{0} \in \mathbb{R}^{p \times q}$ and $D_{1} \in \mathbb{R}^{q \times p}$ are constant matrices. We suppose that $a(x)$ is diagonal 
and partitioned correspondingly as follows:

$$
a(x)=\left(\begin{array}{cc}
a^{-}(x) & 0 \\
0 & a^{+}(x)
\end{array}\right)
$$

where

$$
\begin{aligned}
& a^{-}(x)=\operatorname{diag}\left(\lambda_{1}(x), \lambda_{2}(x), \ldots, \lambda_{p}(x)\right), \\
& a^{+}(x)=\operatorname{diag}\left(\lambda_{p+1}(x), \lambda_{p+2}(x), \ldots, \lambda_{p+q}(x)\right) .
\end{aligned}
$$

Each $\lambda_{i}(x), i=1, \ldots, n$, is a real function of $x$. We assume further the following conditions satisfied for the system (2.1):

H1. The matrices $a(x), b(x)$ are infinitely differentiable functions of $x$ on $[0,1]$ with values in $\mathbb{R}^{n \times n}$ or $a$, $b \in C^{\infty}\left([0,1] ; \mathbb{R}^{n \times n}\right)$.

H2. The first $p$ functions in $a(x)$ are negative, i.e., $\lambda_{i}(x)<0, \forall i=1, \ldots, p, \forall x \in[0,1]$, and the last $q$ functions are positive: $\lambda_{p+i}(x)>0, \forall i=1, \ldots, q, \forall x \in[0,1]$.

H3. The dissipation is true both on the equation and on the boundary: for all $\omega^{-} \in \mathbb{R}^{p}, \omega^{+} \in \mathbb{R}^{q}$ and $x \in[0,1]$,

$$
\begin{gathered}
\tau\left(\begin{array}{c}
\omega^{-} \\
\omega^{+}
\end{array}\right)\left[b(x)+{ }^{\tau} b(x)-\frac{\partial}{\partial x} a(x)\right]\left(\begin{array}{c}
\omega^{-} \\
\omega^{+}
\end{array}\right) \leq 0 \\
\tau \omega^{-}\left[a^{-}(1)+{ }^{\tau} D_{1} a^{+}(1) D_{1}\right] \omega^{-} \leq-r_{1}\left\|\omega^{-}\right\|_{\mathbb{R}^{p}}^{2}, \\
\tau \omega^{+}\left[a^{+}(0)+{ }^{\tau} D_{0} a^{-}(0) D_{0}\right] \omega^{+} \geq r_{2}\left\|\omega^{+}\right\|_{\mathbb{R}^{q}}^{2},
\end{gathered}
$$

where the constants $r_{1} \geq 0$ and $r_{2} \geq 0$ are not both zero, i.e., $\left(r_{1}, r_{2}\right) \neq 0$ and ${ }^{\tau} \omega$ is the transposition of $\omega$.

Generally speaking, the condition $\mathrm{H} 1$ guarantees a good regularity of the solution corresponding to a regular initial data. H2 means that the system is strictly hyperbolic. As illustrated in [40], H2 is not absolutely essential in applications. H3 says that the system is strictly dissipative on the boundary.

Let $C_{0}^{\infty}(0,1)$ denote the space of infinitely differentiable functions of compact support in $(0,1)$. The theorem in [31] can be restated as follows.

Theorem 2.1. For each initial data $R_{0} \in\left(C_{0}^{\infty}(0,1)\right)^{n}$, there is a unique continuously differentiable function $R(x, t)$ which satisfies the four equations in (2.1). Moreover, there exist some positive constants $K$ and $\omega$ such that, for all $R_{0} \in\left(C_{0}^{\infty}(0,1)\right)^{n}$,

$$
\int_{0}^{1}\|R(x, t)\|_{\mathbb{R}^{n}}^{2} \mathrm{~d} x \leq K \mathrm{e}^{-\omega t} \int_{0}^{1}\left\|R_{0}(x)\right\|_{\mathbb{R}^{n}}^{2} \mathrm{~d} x .
$$

Remark 2.1. Since the system (2.1) is linear, for each $t \geq 0$ the mapping $\mathbb{T}(t)$, which associates to the initial data $R_{0} \in\left(C_{0}^{\infty}(0,1)\right)^{n}$ its corresponding solution $R(\cdot, t)$, is linear and bounded from $H=\left(L^{2}(0,1)\right)^{n}$ to $H$. This family of bounded linear operators $\{\mathbb{T}(t)\}_{t \geq 0}$ on $H$ form a semigroup which is strongly continuous from $[0, \infty)$ to $\mathcal{L}(H)$ (called $C^{0}$ semigroup). The Theorem 2.1 says that, for all $R_{0} \in\left(C_{0}^{\infty}(0,1)\right)^{n}$,

$$
\left\|\mathbb{T}(t) R_{0}\right\|_{H}^{2} \leq K \mathrm{e}^{-\omega t}\left\|R_{0}\right\|_{H}^{2}
$$

The subspace $C_{0}^{\infty}(0,1)$ being dense in $L^{2}(0,1)$, it follows that the system is exponentially stable, i.e.,

$$
\|\mathbb{T}(t)\|_{\mathcal{L}(H)} \leq \sqrt{K} \mathrm{e}^{-\omega t / 2}
$$

or, the $C^{0}$ semigroup is exponentially stable. 
Remark 2.2. Numerous hyperbolic PDE systems can be transformed into the form (2.1) (see $[4,32,36])$. In particular the linearized models of the crystallization processes or the ploymerization processes have the form given in $(2.1)$, see $[14,28]$. The constructed Lyapunov functionals, apart from the proof for the essential result, are potentially utilizable for proving stability of nonlinear systems as well as for future control design. A generalization of the essential theorem to nonlinear cases will be exploited in the next section with nonlinearities occurring in the zero order term.

\section{Proof of Theorem 2.1 USING Lyapunov's DiRECT Method}

We assume that $r_{2}>0$. The case $r_{1}>0$ can be dealt with similarly, see the Remark 3.1 below.

The state space for the system $(2.1)$ is the Hilbert space $H=\left(L^{2}(0,1)\right)^{n}$ equipped with the usual inner product: $\|f\|_{H}^{2}=\sum_{k=1}^{n} \int_{0}^{1}\left|f_{k}(x)\right|^{2} \mathrm{~d} x$. Let $H^{1}(0,1)$ be the Sobolev space of $f \in L^{2}(0,1)$ such that $f^{\prime} \in L^{2}(0,1)$, where $f^{\prime}$ denotes the generalized derivative of $f$ with respect to $x$. Define the unbounded linear operator $A$ by

$$
\begin{gathered}
\mathcal{D}(A)=\left\{f=\left(\begin{array}{c}
f^{-} \\
f^{+}
\end{array}\right) \in\left(H^{1}(0,1)\right)^{p+q} \mid f^{-}(0)=D_{0} f^{+}(0), f^{+}(1)=D_{1} f^{-}(1)\right\}, \\
\forall f \in \mathcal{D}(A), \quad A f(x)=a(x) f^{\prime}(x)+b(x) f(x) .
\end{gathered}
$$

Proposition 3.1. For each $R_{0} \in\left(C_{0}^{\infty}(0,1)\right)^{n}$, there is a unique $R(x, t) \in\left(C^{1}([0,1] \times[0, \infty))\right)^{n}$ which satisfies the four equations in (2.1).

Proof of Proposition 3.1. The unbounded operator $A$ defined above is the generator of a $C_{0}$ semigroup of contractions on $H\left(c f\right.$. [39]). Note that $\left(C_{0}^{\infty}(0,1)\right)^{n} \subset \mathcal{D}\left(A^{k}\right), \forall k \in \mathbb{N}$. Hence $R \in\left(C^{\infty}([0,1] \times[0, \infty))\right)^{n}$ (cf. Th. X.8, p. 214, [5]).

We set

$$
W(x)=\left(\begin{array}{cc}
\delta^{-}(x) I_{p} & 0 \\
0 & \delta^{+}(x) I_{q}
\end{array}\right)
$$

where

$$
\delta^{-}(x)=1+\mathrm{e}^{-\theta(1+x)}, \delta^{+}(x)=1-\mathrm{e}^{-\theta(1+x)},
$$

with $\theta>0$. The derivative of $W(x)$ with respect to $x$ is

$$
W^{\prime}(x)=\theta \mathrm{e}^{-\theta(1+x)}\left(\begin{array}{cc}
-I_{p} & 0 \\
0 & I_{q}
\end{array}\right) .
$$

We consider the Lyapunov functional $V_{\theta}: H \rightarrow \mathbb{R}^{+}$such that

$$
V_{\theta}(f)=\int_{0}^{1} \tau f(x) W(x) f(x) \mathrm{d} x .
$$

Lemma 3.1. Let $R(x, t)$ be the solution of $(2.1)$ to the initial data $R_{0} \in\left(C_{0}^{\infty}(0,1)\right)^{n}$. Then there exist some positive constants $c_{1}>0, c_{2}>0$ and $\theta_{1}>0$ such that, for all $\theta \geq \theta_{1}$,

$$
\frac{\mathrm{d} V_{\theta}(R(\cdot, t))}{\mathrm{d} t} \leq-\left(c_{1} \theta-c_{2}\right) \mathrm{e}^{-2 \theta} V_{\theta}(R(\cdot, t)), \quad \forall t \geq 0 .
$$


Proof of Lemma 3.1. We denote by $\dot{f}$ the derivative of $f$ with respect to the time and by $f^{\prime}$ the derivative of $f$ with respect to $x$. We compute $\dot{V}_{\theta}(R(\cdot, t))$ along the trajectory:

$$
\begin{aligned}
\dot{V}_{\theta}(R(\cdot, t))= & 2 \int_{0}^{1}{ }^{\tau} R(x, t) W(x) \dot{R}(x, t) \mathrm{d} x \\
= & 2 \int_{0}^{1} \tau R(x, t) W(x)\left[a(x) R^{\prime}(x, t)+b(x) R(x, t)\right] \mathrm{d} x=\left.{ }^{\tau} R(x, t) W(x) a(x) R(x, t)\right|_{x=0} ^{x=1} \\
& +\int_{0}^{1}{ }^{\tau} R(x, t)\left[W(x) b(x)+{ }^{\tau} b(x) W(x)-(W(x) a(x))^{\prime}\right] R(x, t) \mathrm{d} x .
\end{aligned}
$$

It is sufficient to show that when $\theta>0$ is big, the following holds:

$$
\begin{gathered}
\left.{ }^{\tau} R(x, t) W(x) a(x) R(x, t)\right|_{x=0} ^{x=1} \leq 0, \\
{ }^{\tau} R(x, t)\left[W(x) b(x)+{ }^{\tau} b(x) W(x)-(W(x) a(x))^{\prime}\right] R(x, t) \\
\leq-\left(c_{1} \theta-c_{2}\right) \mathrm{e}^{-\theta(1+x)}{ }^{\tau} R(x, t) W(x) R(x, t) .
\end{gathered}
$$

Indeed, substituting (3.6)-(3.7) into (3.5) gives us the required inequality

$$
\begin{gathered}
\dot{V}_{\theta}(R(\cdot, t)) \leq-\left(c_{1} \theta-c_{2}\right) \int_{0}^{1} \mathrm{e}^{-\theta(1+x)}{ }^{\tau} R(x, t) W(x) R(x, t) \mathrm{d} x \\
\leq-\left(c_{1} \theta-c_{2}\right) \mathrm{e}^{-2 \theta} V_{\theta}(R(\cdot, t)) .
\end{gathered}
$$

We first prove (3.6). By direct computation and by using the boundary conditions in (2.1), it is not difficult to obtain the following identities:

$$
\begin{aligned}
\left.{ }^{\tau} R(x, t) W(x) a(x) R(x, t)\right|_{x=0} ^{x=1}= & { }^{\tau} R(1, t) W(1) a(1) R(1, t)-{ }^{\tau} R(0, t) W(0) a(0) R(0, t) \\
= & { }^{\tau} R^{-}(1, t)\left[a^{-}(1)+{ }^{\tau} D_{1} a^{+}(1) D_{1}\right] R^{-}(1, t) \\
& +\mathrm{e}^{-2 \theta}\left[{ }^{\tau} R^{-}(1, t) a^{-}(1) R^{-}(1, t)-{ }^{\tau} R^{+}(1, t) a^{+}(1) R^{+}(1, t)\right] \\
& -{ }^{\tau} R^{+}(0, t)\left[a^{+}(0)+{ }^{\tau} D_{0} a^{-}(0) D_{0}\right] R^{+}(0, t) \\
& -\mathrm{e}^{-\theta}{ }^{\tau} R^{+}(0, t)\left[{ }^{\tau} D_{0} a^{-}(0) D_{0}-a^{+}(0)\right] R^{+}(0, t) .
\end{aligned}
$$

In the last identity, the first term is negative by $\mathrm{H} 3$, the second term is negative by $\mathrm{H} 2$, the third term is negative by $\mathrm{H} 3$ and the fourth term is positive. However, by H3 there is some constant $\tilde{c}_{1}>0$ such that

$$
\begin{gathered}
-{ }^{\tau} R^{+}(0, t)\left[a^{+}(0)+{ }^{\tau} D_{0} a^{-}(0) D_{0}\right] R^{+}(0, t)-\mathrm{e}^{-\theta}{ }^{\tau} R^{+}(0, t)\left[{ }^{\tau} D_{0} a^{-}(0) D_{0}-a^{+}(0)\right] R^{+}(0, t) \\
\leq-\left[r_{2}-\tilde{c}_{1} \mathrm{e}^{-\theta}\right]\left\|R^{+}(0, t)\right\|_{\mathbb{R}^{q}}^{2} .
\end{gathered}
$$

Hence, there exist some $\theta_{1}>0$ such that (3.6) is true for all $\theta \geq \theta_{1}$.

Now we prove (3.7). Indeed, we write

$$
\begin{aligned}
& W(x) b(x)+{ }^{\tau} b(x) W(x)-(W(x) a(x))^{\prime} \\
& \quad W(x)\left(b(x)+{ }^{\tau} b(x)-a^{\prime}(x)\right)-W^{\prime}(x) a(x)+{ }^{\tau} b(x) W(x)-W(x)^{\tau} b(x) .
\end{aligned}
$$


Set $D(x)=b(x)+{ }^{\tau} b(x)-a^{\prime}(x)$. We have $W(x) D(x)=D(x)+(W(x)-I) D(x)$. Let $\omega \in \mathbb{R}^{n}$. Using H3 we get

$$
\begin{aligned}
{ }^{\tau} \omega W(x) D(x) \omega= & { }^{\tau} \omega D(x) \omega+{ }^{\tau} \omega(W(x)-I) D(x) \omega \\
& \leq \mathrm{e}^{-(1+x) \theta}\|D(x)\|_{\mathcal{L}\left(\mathbb{R}^{n}\right)}\|\omega\|_{\mathbb{R}^{n}}^{2} .
\end{aligned}
$$

It is easy to see that

$$
{ }^{\tau} \omega W(x) D(x) \omega \leq\left[\frac{\|D(x)\|_{\mathcal{L}\left(\mathbb{R}^{n}\right)} \mathrm{e}^{-(1+x) \theta}}{1-\mathrm{e}^{-\theta_{1}}}\right] \tau \omega W(x) \omega .
$$

Using (3.3) we find some constant $\tilde{c}_{2}>0$ such that, for all $\theta \geq \theta_{1}$,

$$
{ }^{\tau} \omega W^{\prime}(x) a(x) \omega \geq \tilde{c}_{2} \theta \mathrm{e}^{-(1+x) \theta} \quad \tau \omega W(x) \omega .
$$

On the other hand, using (3.9) we find some constants $\tilde{c}_{3}>0$ and $\tilde{c}_{4}>0$ such that, for all $\theta \geq \theta_{1}$,

$$
\begin{aligned}
{ }^{\tau} \omega W(x) D(x) \omega & \leq \tilde{c}_{3} \mathrm{e}^{-(1+x) \theta} \tau \omega W(x) \omega, \\
{ }^{\tau} \omega\left[{ }^{\tau} b(x) W(x)-W(x)^{\tau} b(x)\right] \omega & \leq \tilde{c}_{4} \mathrm{e}^{-(1+x) \theta}{ }^{\tau} \omega W(x) \omega .
\end{aligned}
$$

Substituting (3.10)-(3.12) into (3.8) proves directly (3.7). Hence the proof of the Lemma 3.1 is complete.

Remark 3.1. For $r_{1}>0$, we just use $W(x)$ in (3.1) with

$$
\delta^{-}(x)=1-\mathrm{e}^{-2 \theta} \mathrm{e}^{\theta x}, \quad \delta^{+}(x)=1+\mathrm{e}^{-2 \theta} \mathrm{e}^{\theta x} .
$$

The same argument allows to establish the Lemma 3.1.

Proof of Theorem 2.1. The proof is a direct application of the Proposition 3.1 and the Lemma 3.1.

The proof of Theorem 2.1 can be generalized to non-linear systems of the following form:

$$
\left\{\begin{array}{l}
\frac{\partial R}{\partial t}=a(x) \frac{\partial R}{\partial x}+b(x, R) \\
R^{-}(0, t)=D_{0} R^{+}(0, t) \\
R^{+}(1, t)=D_{1} R^{-}(1, t) \\
R(x, 0)=R_{0}(x)
\end{array}\right.
$$

where $b$ is a smooth non-linear function from $(0,1) \times \mathbb{R}^{n}$ to $\mathbb{R}^{n}$ satisfying a non-linear dissipation condition

$$
2{ }^{\tau} \omega b(x, \omega)-{ }^{\tau} \omega a^{\prime}(x) \omega \leq 0, \quad \forall(x, \omega) \in(0,1) \times \mathbb{R}^{n},
$$

and a technical condition like

$$
b(x, 0)=0, \quad \sup _{\substack{x \in(0,1) \\ \omega \in \mathbb{R}^{n}}}\left\|\int_{0}^{1} \frac{\partial b(x, \tau \omega)}{\partial R} \mathrm{~d} \tau\right\|_{\mathcal{L}\left(\mathbb{R}^{n}\right)}<\infty .
$$

Using the Theorem 1.5 (p. 13, [27]) or [13], the above proofs and the identity

$$
b(x, \omega)=\left(\int_{0}^{1} \frac{\partial b(x, \tau \omega)}{\partial R} \mathrm{~d} \tau\right) \omega
$$

we prove the following generalization of Theorem 2.1. 
Corollary 3.1. Suppose that all the hypothesis $\mathrm{H} 1-\mathrm{H} 3$ are satisfied for (3.13) except that the corresponding conditions in $\mathrm{H} 3$ and $\mathrm{H} 1$ are replaced by (3.14) and (3.15), respectively. Then, for every $R_{0} \in C^{1}[0,1]$ satisfying the compatibility condition, the non-linear system (3.13) has a unique solution $R \in C([0, \infty), H)$ and there are some positive constants $K$ and $\alpha$ independent of $R_{0}$ such that:

$$
\int_{0}^{1}\|R(x, t)\|_{\mathbb{R}^{n}}^{2} \mathrm{~d} x \leq K \mathrm{e}^{-\alpha t} \int_{0}^{1}\left\|R_{0}(x)\right\|_{\mathbb{R}^{n}}^{2} \mathrm{~d} x
$$

We will give more comments on Corollary 3.1 with the heat exchanger example in the next section. Notice just that (3.15) is a global Lipschitz condition on the non-linear term which could be improved further, see $[4,21,42]$ and the references therein.

\section{EXAMPLES AND APPLICATIONS}

In this section we work out several examples. One of them is the model of the heat exchanger and another one is a dynamical system described by PDEs defined on the unit disk. We apply directly the Theorem 2.1 to prove the exponential stability of the heat exchanger. For the second example we construct a Lyapunov functional to prove its exponential stability. We show how the Lyapunov direct method is extended to PDE systems of higher spatial dimension (see also $[37,40]$ ). Through these examples we explain how our results are related to the reviewed literature.

Example 1. Consider the contra-current heat exchanger process which is an important apparatus in chemical engineering. The dynamic of the temperatures $T_{1}$ and $T_{2}$ in the contra-current fluids are described by the following PDE:

$$
\left\{\begin{array}{l}
\frac{\partial T_{1}}{\partial t}=-m_{1} \frac{\partial T_{1}}{\partial x}-m_{3}\left(T_{1}-T_{2}\right) \\
\frac{\partial T_{2}}{\partial t}=m_{2} \frac{\partial T_{2}}{\partial x}+m_{4}\left(T_{1}-T_{2}\right) \\
T_{1}(0, t)=T_{10}, \quad T_{2}(1, t)=T_{20} \\
T_{1}(x, 0)=\phi_{1}(x), \quad T_{2}(x, 0)=\phi_{2}(x)
\end{array}\right.
$$

where $m_{i}, i=1,2,3,4$, are positive constants and $T_{10}$ and $T_{20}$ being positive constants represent the inlet temperatures to the heat exchanger. In (4.1), usually $m_{3}$ is different from $m_{4}$. First, this is because the contracurrent fluids may have different physical properties. Second the sectional surfaces may be different for the two fluids even when they have the same physical properties.

It is easy to show that the system (4.1) has a unique stationary solution for each pair $\left(T_{10}, T_{20}\right)$. Using the Theorem 2.1 we prove that for all the initial values $\phi_{1}$ and $\phi_{2}$ of the temperatures, they tend to the stationary ones when the time goes to the infinity.

Let us consider the variations of the temperatures around the stationary ones. The dynamic of the variations is described by the following PDE:

$$
\left\{\begin{array}{l}
\frac{\partial}{\partial t}\left(\begin{array}{l}
R_{1} \\
R_{2}
\end{array}\right)=\left(\begin{array}{cc}
-m_{1} & 0 \\
0 & m_{2}
\end{array}\right) \frac{\partial}{\partial x}\left(\begin{array}{l}
R_{1} \\
R_{2}
\end{array}\right)+\left(\begin{array}{cc}
-m_{3} & m_{3} \\
m_{4} & -m_{4}
\end{array}\right)\left(\begin{array}{l}
R_{1} \\
R_{2}
\end{array}\right), \\
R_{1}(0, t)=0, \quad R_{2}(1, t)=0 \\
R_{1}(x, 0)=\tilde{\phi}_{1}(x), \quad R_{2}(x, 0)=\tilde{\phi}_{2}(x) .
\end{array}\right.
$$


With the linear transformation $R_{1}=\sqrt{m_{3}} \tilde{R}_{1}$ and $R_{2}=\sqrt{m_{4}} \tilde{R}_{2}$, it is not difficult to see that the PDE of $\left(\tilde{R}_{1}, \tilde{R}_{2}\right)$ takes the form (2.1) and satisfies all the hypothesis $\mathrm{H} 1-\mathrm{H} 3$ with

$$
\begin{aligned}
& a(x)=\operatorname{diag}\left(-m_{1}, m_{2}\right), \\
& b(x)=\left(\begin{array}{cc}
-m_{3} & \sqrt{m_{3} m_{4}} \\
\sqrt{m_{3} m_{4}} & -m_{4}
\end{array}\right), \\
& D_{0}=0, \quad D_{1}=0 \text {. }
\end{aligned}
$$

Hence $\left(R_{1}(x, t), R_{2}(x, t)\right)$ tends to zero when $t \rightarrow \infty$. Here the proof of the exponential stability is much simpler than that via its transfer function (see [41]).

Let us consider a non-linear case of (4.1) to show the usefulness of Lyapunov's method in dealing with nonlinearities. The non-linear heat exchanger that we consider is governed by the following PDE

$$
\left\{\begin{array}{l}
\frac{\partial T_{1}}{\partial t}=-m_{1} \frac{\partial T_{1}}{\partial x}-m_{3} \phi\left(T_{1}-T_{2}\right) \\
\frac{\partial T_{2}}{\partial t}=m_{2} \frac{\partial T_{2}}{\partial x}+m_{4} \phi\left(T_{1}-T_{2}\right) \\
T_{1}(0, t)=0, \quad T_{2}(1, t)=0 \\
T_{1}(x, 0)=\varphi_{1}(x), \quad T_{2}(x, 0)=\varphi_{2}(x)
\end{array}\right.
$$

where $\phi$ is a smooth increasing function such that $\phi(0)=0$ and $\sup _{\xi \in \mathbb{R}}\left|\phi^{\prime}(\xi)\right| \leq M_{1}$ for some constant $M_{1}>0$. We claim that for every smooth initial condition $\left(\varphi_{1}, \varphi_{2}\right)$, the solution $\left(T_{1}(\cdot, t), T_{2}(\cdot, t)\right)$ converges exponentially to zero in $\left(L^{2}(0,1)\right)^{2}$ as the time goes to the infinity. Indeed, as in the linear case we use the transformation $R_{1}=T_{1} / \sqrt{m_{3}}$ and $R_{2}=T_{2} / \sqrt{m_{4}}$ to rewrite (4.3) as follows

$$
\left\{\begin{array}{l}
\frac{\partial R_{1}}{\partial t}=-m_{1} \frac{\partial R_{1}}{\partial x}-\sqrt{m_{3}} \phi\left(\sqrt{m_{3}} R_{1}-\sqrt{m_{4}} R_{2}\right) \\
\frac{\partial R_{2}}{\partial t}=m_{2} \frac{\partial R_{2}}{\partial x}+\sqrt{m_{4}} \phi\left(\sqrt{m_{3}} R_{1}-\sqrt{m_{4}} R_{2}\right) \\
R_{1}(0, t)=0, \quad R_{2}(1, t)=0 \\
R_{1}(x, 0)=\varphi_{1}(x) / \sqrt{m_{3}}, \quad R_{2}(x, 0)=\varphi_{2}(x) / \sqrt{m_{4}}
\end{array}\right.
$$

All the conditions of Corollary 3.1 are satisfied on the system (4.4). By applying Corollary 3.1 we prove exponential stability of the non-linear heat exchanger. By direct computation with the Lyapunov functional defined in (3.4) and applied on (4.4), we can find some positive constants $k_{1}$ and $k_{2}$ such that for every smooth solution $R(x, t)$,

$$
\frac{\mathrm{d} V_{\theta}(R(\cdot, t))}{\mathrm{d} t} \leq-\left(\theta k_{1}-k_{2}\right) \mathrm{e}^{-2 \theta} V_{\theta}(R(\cdot, t)) .
$$

This also implies exponential stability of (4.3).

Remark 4.1. The matrix $B=\left[\begin{array}{cc}-m_{3} & m_{3} \\ m_{4} & -m_{4}\end{array}\right]$ in the system (4.2) has for eigenvalues $\lambda_{1}=0$ and $\lambda_{2}=$ $-\left(m_{3}+m_{4}\right)$. Through a transformation the system is written in the conservative-dissipative form

$$
R_{t}=\frac{1}{m_{3}+m_{4}}\left[\begin{array}{cc}
m_{3} m_{2}-m_{4} m_{1} & m_{3} m_{4}\left(m_{1}+m_{2}\right) \\
m_{1}+m_{2} & m_{2} m_{4}-m_{1} m_{3}
\end{array}\right] R_{x}+\left[\begin{array}{cc}
0 & 0 \\
0 & -\left(m_{3}+m_{4}\right)
\end{array}\right] R .
$$

(We denote, sometimes by $R_{t}, R$ 's partial derivative of $t$.) It is easy to verify that the system is strictly entropy dissipative (w.r.t. the entropy $\mathcal{E}(R)={ }^{\tau} R R$ ) and satisfies the Shizuta-Kawashima condition. From [4], Theorem 4.2, for any initial condition $R^{0} \in L^{1} \cap L^{2}\left(\mathbb{R}, \mathbb{R}^{2}\right)$ the solution of (4.5) decays to zero in the $L^{2}$ norm with the rate $\mathcal{O}\left(t^{-\frac{1}{4}}\right)$ as $t \rightarrow \infty$. Notice that $(x, t) \in \mathbb{R} \times \mathbb{R}^{+}$in (4.5). 
Example 2. Let $\Omega$ be the open unit disk in the plane: $\Omega=\left\{(x, y) \in \mathbb{R}^{2} \mid x^{2}+y^{2}<1\right\}$. We consider the following PDE

$$
\partial_{t}\left[\begin{array}{l}
u_{1} \\
u_{2}
\end{array}\right]=\left[\begin{array}{l}
\partial_{x} u_{2}+\partial_{y} u_{1} \\
\partial_{x} u_{1}-\partial_{y} u_{2}-2 u_{2}
\end{array}\right],(x, y) \in \Omega, \quad t \in(0, \infty)
$$

satisfying the boundary conditions:

$$
\left\{\begin{array}{l}
(y+1) u_{1}+x u_{2}=0, \\
x u_{1}+(1-y) u_{2}=0,
\end{array} \quad(x, y) \in \partial \Omega\right.
$$

and the initial condition

$$
u(x, y, 0)=u^{0}(x, y) .
$$

Let the state space for the system (4.6)-(4.7) be the Hilbert space $X=\left(L^{2}(\Omega)\right)^{2}$ equipped with the norm: $\|u\|_{X}^{2}=\int_{\Omega}\left[\left|u_{1}(x, y)\right|^{2}+\left|u_{2}(x, y)\right|^{2}\right] \mathrm{d} x \mathrm{~d} y$. Define the unbounded linear operator $A$ by

$$
\begin{gathered}
D(A)=\left\{u=\left[\begin{array}{l}
u_{1} \\
u_{2}
\end{array}\right] \in\left(C^{1}(\Omega) \cap C(\bar{\Omega})\right)^{2} \mid \begin{array}{l}
(y+1) u_{1}+x u_{2}=0, \\
x u_{1}+(1-y) u_{2}=0,
\end{array},(x, y) \in \partial \Omega\right\} \\
A u=\left[\begin{array}{l}
\partial_{x} u_{2}+\partial_{y} u_{1} \\
\partial_{x} u_{1}-\partial_{y} u_{2}-2 u_{2}
\end{array}\right], \quad \forall u \in D(A) .
\end{gathered}
$$

Lemma 4.1. The linear operator $A$ is closable, i.e., it has a closed extension, noted by $\tilde{A}$ and called closure of $A$.

Proof of Lemma 4.1. It suffices to prove the assertion that for each sequence $\left(f_{n}\right)$ in $D(A)$,

$$
\left(f_{n} \rightarrow 0, A f_{n} \rightarrow \xi \text { in } X\right) \Rightarrow(\xi=0) .
$$

Indeed, for any $g \in\left(C_{0}^{\infty}(\Omega)\right)^{2}$,

$$
\begin{aligned}
\left(A f_{n}, g\right) & =\int_{\Omega}\left(g_{1} \partial_{x} f_{n, 2}+g_{1} \partial_{y} f_{n, 1}+g_{2} \partial_{x} f_{n, 1}-g_{2} \partial_{y} f_{n, 2}-2 g_{2} f_{n, 2}\right) \mathrm{d} x \mathrm{~d} y \\
& =-\int_{\Omega}\left(f_{n, 2} \partial_{x} g_{1}+f_{n, 1} \partial_{y} g_{1}+f_{n, 1} \partial_{x} g_{2}-f_{n, 2} \partial_{y} g_{2}+2 g_{2} f_{n, 2}\right) \mathrm{d} x \mathrm{~d} y
\end{aligned}
$$

where $(\cdot, \cdot)$ denotes the inner product on $X$. By the hypothesis in (4.11), we see that the right member in (4.12) converges to 0 as $n$ goes to infinity. By the density of $C_{0}^{\infty}(\Omega)$ in $L^{2}(\Omega)$ and the continuity of the inner product, we deduce

$$
\forall g \in X, \quad(\xi, g)=\left(\lim _{n} A f_{n}, g\right)=0 .
$$

Consequently $\xi=0$. This completes the proof.

It is easy to see that the domain of the closure $D(\tilde{A})$ is given by

$$
D(\tilde{A})=\left\{u=\left[\begin{array}{l}
u_{1} \\
u_{2}
\end{array}\right] \in\left(H^{1}(\Omega)\right)^{2} \mid \begin{array}{l}
(y+1) u_{1}+x u_{2}=0, \\
x u_{1}+(1-y) u_{2}=0,
\end{array} \text { a.e. }(x, y) \in \partial \Omega\right\} .
$$

Proposition 4.1. For each initial data $u^{0} \in\left(C_{0}^{\infty}(\Omega)\right)^{2}$, there exists a unique solution $u \in C^{1}(\bar{\Omega} \times[0, \infty))$ which satisfies the system (4.6)-(4.8). Moreover this solution decays exponentially to zero in $X$ norm as time goes to infinity. 
Proof of Proposition 4.1. We prove that the operator $\tilde{A}$ is the generator of a $C^{0}$ semigroup of contractions which is exponentially stable on $X$.

We prove first that $\tilde{A}$ is dissipative. Formally, we can write the operator $A$ under the form:

$$
A=L_{1}(x, y) \partial_{x}+L_{2}(x, y) \partial_{y}+\Sigma(x, y),
$$

where

$$
L_{1}(x, y)=\left(\begin{array}{ll}
0 & 1 \\
1 & 0
\end{array}\right), \quad L_{2}(x, y)=\left(\begin{array}{ll}
1 & 0 \\
0 & -1
\end{array}\right), \quad \Sigma(x, y)=\left(\begin{array}{ll}
0 & 0 \\
0 & -2
\end{array}\right) .
$$

The boundary conditions (4.7) are equivalent to

$$
u(x, y) \in S(x, y)=\operatorname{Ker}(\Lambda(x, y)), \forall(x, y) \in \partial \Omega
$$

where

$$
\Lambda(x, y)=\left(\begin{array}{ll}
1+y & x \\
x & 1-y
\end{array}\right) .
$$

Since the determinant of $\Lambda(x, y)$ is zero on $\partial \Omega$, then $\operatorname{dim}(S(x, y))=1$ for all $(x, y) \in \partial \Omega$.

Let $u \in D(A)$, by the use of the divergence theorem we get

$$
\begin{aligned}
(A u, u) & =\int_{\Omega}\left(u_{1} \partial_{x} u_{2}+u_{1} \partial_{y} u_{1}+u_{2} \partial_{x} u_{1}-u_{2} \partial_{y} u_{2}-2 u_{2}^{2}\right) \mathrm{d} x \mathrm{~d} y \\
& =\frac{1}{2} \int_{\partial \Omega}\left(y u_{1}^{2}+2 x u_{1} u_{2}-y u_{2}^{2}\right) \mathrm{d} \sigma-2 \int_{\Omega} u_{2}^{2} \mathrm{~d} x \mathrm{~d} y \\
& =\frac{1}{2} \int_{\partial \Omega}\left(\begin{array}{ll}
u_{1} & u_{2}
\end{array}\right)\left(\begin{array}{cc}
y & x \\
x & -y
\end{array}\right)\left(\begin{array}{l}
u_{1} \\
u_{2}
\end{array}\right) \mathrm{d} \sigma-2 \int_{\Omega} u_{2}^{2} \mathrm{~d} x \mathrm{~d} y .
\end{aligned}
$$

From the boundary conditions (4.7), it comes

$$
\left(\begin{array}{l}
u_{1} \\
u_{2}
\end{array}\right)=\left(\begin{array}{cc}
-y & -x \\
-x & y
\end{array}\right)\left(\begin{array}{l}
u_{1} \\
u_{2}
\end{array}\right)
$$

Replacing (4.15) in (4.14), we get

$$
(A u, u)=-\frac{1}{2} \int_{\partial \Omega}\left(u_{1}^{2}+u_{2}^{2}\right) \mathrm{d} \sigma-2 \int_{\Omega} u_{2}^{2} \mathrm{~d} x \mathrm{~d} y \leq 0,
$$

so the operator $A$ is dissipative in $X$. Therefore $\tilde{A}$ is also dissipative.

Formally, the computation in (4.14)-(4.16) leads to the following non-positive matrix:

$$
\Sigma(x, y)+\Sigma^{*}(x, y)-\partial_{x} L_{1}(x, y)-\partial_{y} L_{2}(x, y) \leq 0, \forall(x, y) \in \Omega
$$

hence $A$ is formally dissipative, and

$$
u^{*} l(x, y) u \leq 0, \forall u \in S(x, y), \forall(x, y) \in \partial \Omega .
$$

In (4.17), $l(x, y)=n_{1}(x, y) L_{1}(x, y)+n_{2}(x, y) L_{2}(x, y)$ is called the boundary matrix and $n(x, y)=\left(n_{1}(x, y)\right.$; $\left.n_{2}(x, y)\right)=(x ; y)$ is the outer unit normal to $\Omega$ at $(x, y) \in \partial \Omega$. Moreover the subspace $S(x, y)$ of $\mathbb{R}^{2}$ is maximal for the property (4.17), because $\operatorname{dim}(S(x, y))=1$ and $\mathbb{R}^{2}$ does not verify it. 
Next we prove that $I-\tilde{A}$ is surjective. Given $g \in X$ we look for some $f \in D(\tilde{A})$ such that

$$
(I-\tilde{A}) f=g .
$$

From Lax and Philipps (see [20], Prop. 3.2) we know that for each $g \in X$, there exists some strongly converging sequence $\left(f_{n}\right)$ in $D(A)$ such that $(I-A) f_{n}$ converges strongly to $g$. By definition the limit $f$ of $\left(f_{n}\right)$ belongs to $D(\tilde{A})$ and satisfies (4.18). Hence $I-\tilde{A}$ is surjective. We have proven that $\tilde{A}$ is a generator of a $C^{0}$ semigroup of contractions $T(t)$ on $X$.

It remains to prove the exponential stability of $\tilde{A}$. For this purpose we consider the Lyapunov functional

$$
V(u)=\int_{\Omega} \mathrm{e}^{y}\left(\left|u_{1}(x, y, t)\right|^{2}+\left|u_{2}(x, y, t)\right|^{2}\right) \mathrm{d} x \mathrm{~d} y,
$$

for $u(t)=T(t) u^{0}$ a solution from the initial condition $u^{0} \in D(A)$.

By differentiating the function $V(u(t))$ along the trajectory of (4.6)-(4.8) and by similar computation as above, we get

$$
\dot{V}(u) \leq-V(u) .
$$

Consequently the semigroup is exponentially stable: there exists a constant $M \geq 1$ such that

$$
\|T(t)\|_{\mathcal{L}(X)} \leq M \mathrm{e}^{-t} .
$$

Hence the proof is complete.

Remark 4.2. By direct algebraic computations from (4.6), we find that both $u_{1}$ and $u_{2}$ satisfy the classical D'Alembert equations as follows:

$$
\begin{aligned}
& u_{1 t t}=\Delta u_{1}-2 u_{1 t}+2 u_{1 y} \\
& u_{2 t t}=\Delta u_{2}-2 u_{2 t}+2 u_{2 y}
\end{aligned}
$$

which are coupled via the boundary condition (4.7). Nevertheless it does not seem possible to deduce, from the result of [2], that the total energy in this case decays to zero as $t \rightarrow \infty$. Indeed, by a transformation $\tilde{u}_{1}=\mathrm{e}^{y} u_{1}$ and $\tilde{u}_{2}=\mathrm{e}^{y} u_{2}$ the system (4.19) with (4.7) is written as

$$
\begin{aligned}
& \tilde{u}_{1 t t}=\Delta \tilde{u}_{1}-\tilde{u}_{1}-2 \tilde{u}_{1 t} \\
& \tilde{u}_{2 t t}=\Delta \tilde{u}_{2}-\tilde{u}_{2}-2 \tilde{u}_{2 t}, \quad \forall(x, y, t) \in \Omega \times \mathbb{R}^{+}
\end{aligned}
$$

which is supplemented by homogeneous boundary condition

$$
\left\{\begin{array}{l}
(y+1) \tilde{u}_{1}+x \tilde{u}_{2}=0 \\
x \tilde{u}_{1}+(1-y) \tilde{u}_{2}=0, \quad(x, y, t) \in \partial \Omega \times \mathbb{R}^{+} .
\end{array}\right.
$$

Both $\tilde{u}_{1}$ and $\tilde{u}_{2}$ satisfy the damped wave equation (4.20) and their energies are given by $E\left(u_{i}\right)=\int_{\Omega}\left(u_{i t}^{2}+\right.$ $\left.\left\|\nabla u_{i}\right\|^{2}+u_{i}^{2}\right) \mathrm{d} x \mathrm{~d} y, i=1,2$. However the boundary condition (4.21) is not of the Dirichlet type. To the best of our knowledge it is still an open question to prove the stability of the system (4.20)-(4.21) in the energy space defined by $V(\tilde{u})=E\left(\tilde{u}_{1}\right)+E\left(\tilde{u}_{2}\right)$.

Remark 4.3. From the above proof of Proposition 4.1 the system (4.6) with the Dirichlet boundary condition has a unique equilibrium solution $u=(0,0)$ which is exponentially stable in $X$ :

$$
\begin{aligned}
& u_{1 t}=u_{2 x}+u_{1 y} \\
& u_{2 t}=u_{1 x}-u_{2 y}-2 u_{2}, \quad(x, y, t) \in \Omega \times \mathbb{R}^{+} \\
& u_{1}=u_{2}=0, \quad(x, y, t) \in \partial \Omega \times \mathbb{R}^{+} \\
& u(x, y, 0)=u^{0}(x, y), \quad(x, y) \in \Omega .
\end{aligned}
$$


Further the exponential stability of the null equilibrium solution is true for the system (4.22) defined on a more general bounded open domain $\Omega \subset \mathbb{R}^{2}$. It is interesting to note that, in the case of Dirichlet boundary condition, the energy $V(u)$ of every smooth solution of (4.22) converges exponentially to zero with time going to infinity, by direct application of [31], Theorem 2. Indeed, in this case the transformed system is written as

$$
\begin{aligned}
& \tilde{u}_{1 t t}=\Delta \tilde{u}_{1}-\tilde{u}_{1}-2 \tilde{u}_{1 t} \\
& \tilde{u}_{2 t t}=\Delta \tilde{u}_{2}-\tilde{u}_{2}-2 \tilde{u}_{2 t}, \quad(x, y, t) \in \Omega \times \mathbb{R}^{+} \\
& \tilde{u}_{1}=\tilde{u}_{2}=0, \quad(x, y, t) \in \partial \Omega \times \mathbb{R}^{+}, \\
& \tilde{u}(x, y, 0), \tilde{u}_{t}(x, y, 0) \text { known for }(x, y) \in \Omega .
\end{aligned}
$$

Define $A=-\Delta+I$ which is strictly positive and self-adjoint in $L^{2}(\Omega)$. Let $H$ be the Hilbert space $\mathcal{D}\left(A^{1 / 2}\right) \oplus L^{2}(\Omega)$ with norm $\|u\|_{H}^{2}=\left\|A^{1 / 2} u_{1}\right\|_{L^{2}}^{2}+\left\|u_{2}\right\|_{L^{2}}^{2}$. There exist some positive constants $c>0$ and $\alpha>0$ such that $\left\|\left(\tilde{u}_{i}, \tilde{u}_{i t}\right)\right\|_{H} \leq c \mathrm{e}^{-\alpha t}\left\|\left(\tilde{u}_{i}^{0}, \tilde{u}_{i t}^{0}\right)\right\|_{H}, i=1,2$.

Remark 4.4. The exponential stability of the system (4.23) can be also deduced directly from that of (4.22). Indeed, each function $u, u_{t}, u_{x}$ or $u_{y}$ satisfies the same PDE in (4.22) and the Dirichlet condition: $u_{i}=u_{i t}=$ $u_{i x}=u_{i y}=0, \forall i=1,2$, on $\partial \Omega \times \mathbb{R}^{+}$. From Remark 4.3 there are positive constants $M>0$ and $\omega>0$ such that $\|u\|_{L^{2}} \leq M \mathrm{e}^{-\omega t}\left\|u^{0}\right\|_{L^{2}}$ and $\left\|u_{s}\right\|_{L^{2}} \leq M \mathrm{e}^{-\omega t}\left\|u_{s}^{0}\right\|_{L^{2}}, \forall s=t, x, y$. Addition of these four inequalities gives the required inequality $V(u(t)) \leq c \mathrm{e}^{-\alpha t} V\left(u^{0}\right)$ for some positive constants $c, \alpha$.

Example 3. We consider the following first order hyperbolic PDE system which describes in particular dynamical behaviour of plug flow reactors:

$$
\left\{\begin{array}{l}
\frac{\partial R(x, t)}{\partial t}=-\frac{\partial R(x, t)}{\partial x}+N(R(x, t)), \quad x \in(0,1), t>0 \\
R(0, t)=0, \quad R(x, 0)=R^{0}(x)
\end{array}\right.
$$

where $R(x, t) \in \mathbb{R}^{n}$. We suppose that $N(z)$ is a continuously differentiable function defined on $\mathbb{R}^{n}$ such that $N(0)=0$ and $\|N(z)\|_{\mathbb{R}^{n}} \leq L\|z\|_{\mathbb{R}^{n}}$ with some Lipschitz constant $L>0$. The state space is the Hilbert space $X=\left(L^{2}(0,1)\right)^{n}$ equipped with the inner product $\|f\|_{X}^{2}=\int_{0}^{1}\|f(x)\|_{\mathbb{R}^{n}}^{2} \mathrm{~d} x$. Then the trivial solution $R=0$ is an exponentially stable equilibrium solution of the system (4.24).

Theorem 4.1. Suppose that $N: \mathbb{R}^{n} \rightarrow \mathbb{R}^{n}$ is Lipschitz continuous with Lipschitz constant $L>0$. Then there exist positive constants $M$ and $\omega$ such that for every smooth $R^{0}(x)$ with $R^{0}(0)=0$,

$$
\|R(\cdot, t)\|_{X} \leq M \mathrm{e}^{-\omega t}\left\|R^{0}\right\|_{X}
$$

Proof of Theorem 4.1. From [27], Theorem 1.5, p. 187, for each initial condition $R^{0} \in C^{1}[0,1]$ such that $R^{0}(0)=0$ the solution $R(x, t)$ is a classical solution of the system (4.24). Consider the Lyapunov functional candidate $V: X \rightarrow \mathbb{R}^{+}$such that $V(f)=\int_{0}^{1} \mathrm{e}^{-\alpha x}\|f(x)\|_{X}^{2} \mathrm{~d} x$. Differentiating the function $V(R(\cdot, t))$ along the trajectory of $(4.24)$ we get

$$
\begin{aligned}
\dot{V}(R(\cdot, t)) & =2 \int_{0}^{1} \mathrm{e}^{-\alpha x} R^{\top}(x, t) \partial_{t} R(x, t) \mathrm{d} x \\
& =2 \int_{0}^{1} \mathrm{e}^{-\alpha x} R^{\top}(x, t)\left[-\partial_{x} R(x, t)+N(R(x, t))\right] \mathrm{d} x \\
& =-\left.\mathrm{e}^{-\alpha x}\|R(x, t)\|_{\mathbb{R}^{n}}^{2}\right|_{0} ^{1}-\alpha V(R(\cdot, t))+2 \int_{0}^{1} \mathrm{e}^{-\alpha x} R^{\top}(x, t) N(R(x, t)) \mathrm{d} x .
\end{aligned}
$$


Using the global Lipschitz condition on the nonlinearity and the above identity we deduce that

$$
\begin{array}{r}
\dot{V}(R(\cdot, t)) \leq-\mathrm{e}^{-\alpha}\|R(1, t)\|_{\mathbb{R}^{n}}^{2}-\alpha V(R(\cdot, t))+2 \int_{0}^{1} \mathrm{e}^{-\alpha x} L\|R(x, t)\|_{\mathbb{R}^{n}}^{2} \mathrm{~d} x \\
\leq-(\alpha-2 L) V(R(\cdot, t)) .
\end{array}
$$

Taking $\alpha \geq 3 L$ in (4.26), we obtain the following inequality

$$
V(R(\cdot, t)) \leq \mathrm{e}^{-L t} V\left(R^{0}\right)
$$

Since the square root of $V(f)$ defines an equivalent norm of $X: C_{1}\|f\|_{X}^{2} \leq V(f) \leq C_{2}\|f\|_{X}^{2}$ for some positive constants $C_{1}$ and $C_{2}$, the inequality (4.27) implies existence of some positive constants $M$ and $\omega$ such that (4.25) holds.

\section{EXPONENTIAL STABILITY FOR THE UNIFORM TOPOLOGY}

The Theorem 2.1 states the exponential stability for the $L^{2}$ norm. In some particular cases, we can establish an exponential stability result for the uniform topology by extending the Lyapunov method to the Banach spaces $L^{r}(1<r<+\infty)$. We will work on a previous example and we will use the framework of one-parameter semigroups.

\subsection{Exponential stability in Banach spaces $L^{r}, 1<r<\infty$}

Consider a fixed real number $1<r<+\infty$ and the Banach space $X_{r}=\left(L^{r}(0,1)\right)^{2}$ equipped with the norm $\|f\|_{r}^{2}=\sum_{i=1}^{2}\left(\int_{0}^{1}\left|f_{i}(\xi)\right|^{r} \mathrm{~d} \xi\right)^{\frac{2}{r}}$. Let's consider the PDE

$$
\left\{\begin{array}{l}
\frac{\partial}{\partial t}\left(\begin{array}{l}
R_{1} \\
R_{2}
\end{array}\right)=\left(\begin{array}{cc}
-m_{1} & 0 \\
0 & m_{2}
\end{array}\right) \frac{\partial}{\partial x}\left(\begin{array}{l}
R_{1} \\
R_{2}
\end{array}\right)+\left(\begin{array}{cc}
-m_{3} & \sqrt{m_{3} m_{4}} \\
\sqrt{m_{3} m_{4}} & -m_{4}
\end{array}\right)\left(\begin{array}{l}
R_{1} \\
R_{2}
\end{array}\right) \\
R_{1}(0, t)=0, \quad R_{2}(1, t)=0 \\
R_{1}(x, 0)=\tilde{\phi}_{1}(x), \quad R_{2}(x, 0)=\tilde{\phi}_{2}(x)
\end{array}\right.
$$

We put this PDE in the form of abstract Cauchy problem

$$
\left\{\begin{array}{l}
\dot{u}(t)=A_{r} u(t)+B_{r} u(t) \\
u(0)=\left(\tilde{\phi}_{1}, \tilde{\phi}_{2}\right)
\end{array}\right.
$$

for the vector-valued function $u: \mathbb{R}^{+} \rightarrow X_{r}$. We define the operators $A_{r}, B_{r}$ on $X_{r}$

$$
\begin{aligned}
& A_{r} f=\left(\begin{array}{ll}
-m_{1} & 0 \\
0 & m_{2}
\end{array}\right) f^{\prime}, \quad D\left(A_{r}\right)=\left\{f \in\left(W^{1, r}(0,1)\right)^{2} \mid f_{1}(0)=f_{2}(1)=0\right\} \\
& B_{r} f=\left(\begin{array}{cc}
-m_{3} & \sqrt{m_{3} m_{4}} \\
\sqrt{m_{3} m_{4}} & -m_{4}
\end{array}\right) f, \quad D\left(B_{r}\right)=X_{r} .
\end{aligned}
$$


Let $\rho\left(A_{r}\right)$ and $R\left(\lambda ; A_{r}\right)$ denote the resolvent set and the resolvent operator of $A_{r}$, respectively. It is easy to see that $\rho\left(A_{r}\right) \supset(0, \infty)$ and, for all $\lambda \in(0, \infty), x \in[0,1]$ and $\varphi \in X_{r}$,

$$
R\left(\lambda ; A_{r}\right) \varphi(x)=\left(\begin{array}{c}
\frac{1}{m_{1}} \int_{0}^{x} \mathrm{e}^{\frac{\lambda}{m_{1}}(\xi-x)} \varphi_{1}(\xi) \mathrm{d} \xi \\
-\frac{1}{m_{2}} \int_{1}^{x} \mathrm{e}^{\frac{\lambda}{m_{2}}(x-\xi)} \varphi_{2}(\xi) \mathrm{d} \xi
\end{array}\right) .
$$

By the well-known convolution inequality we prove that $\left\|R\left(\lambda ; A_{r}\right) \varphi\right\| \leq\|\varphi\| / \lambda$ for all $\lambda>0$ and all $\varphi \in X_{r}$. It follows that $A_{r}$ generates a $C^{0}$ semigroup $\left(S_{r}(t)\right)_{t \geq 1}$ of contractions on $X_{r}$. So, by the bounded perturbation $A_{r}+B_{r}$ generates also a $C^{0}$ semigroup $\left(T_{r}(t)\right)_{t \geq 1}$ on $X_{r}$. Moreover $T_{r}(t)$ is a semigroup of contractions on $X_{r}$ because the bounded operator $B_{r}$ is dissipative. Indeed we prove that the semigroup $U_{r}(t)$ generated by $B_{r}$ is a semigroup of contractions through a Lyapunov functional.

Let $\phi^{0} \in X_{r}$ and $\phi(t)=\mathrm{e}^{t B_{r}} \phi^{0}$. Consider the Lyapunov functional

$$
W(\phi(t))=\|\phi\|_{r}^{2}=\left(\int_{0}^{1}\left|\phi_{1}(x, t)\right|^{r} \mathrm{~d} x\right)^{\frac{2}{r}}+\left(\int_{0}^{1}\left|\phi_{2}(x, t)\right|^{r} \mathrm{~d} x\right)^{\frac{2}{r}} .
$$

Differentiating $W(\phi(t))$ gives:

$$
\begin{aligned}
\frac{1}{2} \dot{W}(\phi(t))= & -m_{3}\left(\int_{0}^{1}\left|\phi_{1}(x, t)\right|^{r} \mathrm{~d} x\right)^{\frac{2}{r}}-m_{4}\left(\int_{0}^{1}\left|\phi_{2}(x, t)\right|^{r} \mathrm{~d} x\right)^{\frac{2}{r}} \\
& +\sqrt{m_{3} m_{4}}\left[\left(\int_{0}^{1}\left|\phi_{1}(x, t)\right|^{r} \mathrm{~d} x\right)^{\frac{2-r}{r}} \int_{0}^{1}\left|\phi_{1}(x, t)\right|^{r-1} \operatorname{sign}\left(\phi_{1}\right) \phi_{2}(x, t) \mathrm{d} x\right. \\
& \left.+\left(\int_{0}^{1}\left|\phi_{2}(x, t)\right|^{r} \mathrm{~d} x\right)^{\frac{2-r}{r}} \int_{0}^{1}\left|\phi_{2}(x, t)\right|^{r-1} \operatorname{sign}\left(\phi_{2}\right) \phi_{1}(x, t) \mathrm{d} x\right]
\end{aligned}
$$

By applying Hölder inequality in the two integrals in brackets, we get easily

$$
\frac{1}{2} \dot{W}(\phi(t)) \leq\left(\left\|\phi_{1}\right\|_{r},\left\|\phi_{2}\right\|_{r}\right)\left(\begin{array}{cc}
-m_{3} & \sqrt{m_{3} m_{4}} \\
\sqrt{m_{3} m_{4}} & -m_{4}
\end{array}\right)\left(\begin{array}{c}
\left\|\phi_{1}\right\|_{r} \\
\left\|\phi_{2}\right\|_{r}
\end{array}\right) \leq 0
$$

This inequality implies that $\left\|\mathrm{e}^{t B_{r}} \phi^{0}\right\|_{r} \leq\left\|\phi^{0}\right\|_{r}$. Therefore $B_{r}$ is dissipative and $A_{r}+B_{r}$ generates a $C^{0}$ semigroup of contractions.

To prove the exponential stability of $T_{r}(t)$, we will construct a Lyapunov functional. Let us consider $\phi^{0} \in$ $D\left(A_{r}\right), \phi(t)=T_{r}(t) \phi^{0}$ is solution of the initial value problem:

$$
\left\{\begin{array}{l}
\dot{\phi}(t)=\left(A_{r}+B_{r}\right) \phi(t) \\
\phi(0)=\phi^{0}
\end{array}\right.
$$

Consider the Lyapunov functional

$$
V_{\theta}(\phi(t))=\left(\int_{0}^{1} \mathrm{e}^{\theta r x}\left|\phi_{1}(x, t)\right|^{r} \mathrm{~d} x\right)^{\frac{2}{r}}+\left(\int_{0}^{1} \mathrm{e}^{\theta r x}\left|\phi_{2}(x, t)\right|^{r} \mathrm{~d} x\right)^{\frac{2}{r}},
$$

where $\theta$ denotes a real number. 
To simplify the notations we set

$$
\left\|\left|\phi_{i} \|\right|=\left(\int_{0}^{1} \mathrm{e}^{\theta r x}\left|\phi_{i}(x, t)\right|^{r} \mathrm{~d} x\right)^{\frac{1}{r}}, i=1,2 .\right.
$$

By direct computation we find the derivative of $V_{\theta}(\phi(t))$ with respect to $t$ as follows:

$$
\begin{aligned}
& \frac{1}{2} \dot{V}_{\theta}(\phi(t))=\frac{1}{r}\left\|| \phi _ { 1 } \| | ^ { 2 - r } \left\{-m_{1} \mathrm{e}^{\theta r}\left|\phi_{1}(1, t)\right|^{r}+r \theta m_{1} \int_{0}^{1} \mathrm{e}^{\theta r x}\left|\phi_{1}(x, t)\right|^{r} \mathrm{~d} x\right.\right. \\
& \left.+\int_{0}^{1} r \mathrm{e}^{\theta r x}\left|\phi_{1}(x, t)\right|^{r-1} \operatorname{sign}\left(\phi_{1}(x, t)\right)\left[-m_{3} \phi_{1}(x, t)+\sqrt{m_{3} m_{4}} \phi_{2}(x, t)\right] \mathrm{d} x\right\} \\
& \quad+\frac{1}{r}\left\|| \phi _ { 2 } \| | ^ { 2 - r } \left\{-m_{2}\left|\phi_{2}(0, t)\right|^{r}-r \theta m_{2} \int_{0}^{1} \mathrm{e}^{\theta r x}\left|\phi_{2}(x, t)\right|^{r} \mathrm{~d} x\right.\right. \\
& \left.+\int_{0}^{1} r \mathrm{e}^{\theta r x}\left|\phi_{2}(x, t)\right|^{r-1} \operatorname{sign}\left(\phi_{2}(x, t)\right)\left[\sqrt{m_{3} m_{4}} \phi_{1}(x, t)-m_{4} \phi_{2}(x, t)\right] \mathrm{d} x\right\} .
\end{aligned}
$$

Using the Hölder inequality we obtain the following estimate:

$$
\frac{1}{2} \dot{V}_{\theta}(\phi(t)) \leq-\left(\left\|\left|\phi _ { 1 } \left\|\left|,\left\|\left|\phi_{2} \|\right|\right) \Lambda_{\theta}\left(\begin{array}{c}
\left\|\left|\phi_{1} \|\right|\right. \\
\left\|\left|\phi_{2} \|\right|\right.
\end{array}\right)\right.\right.\right.\right.\right.
$$

where the matrix $\Lambda_{\theta}$ is symmetric and defined by

$$
\Lambda_{\theta}=\left(\begin{array}{cc}
m_{3}-\theta m_{1} & -\sqrt{m_{3} m_{4}} \\
-\sqrt{m_{3} m_{4}} & m_{4}+\theta m_{2}
\end{array}\right)
$$

Proposition 5.1. Assume that $m_{2} m_{3}-m_{1} m_{4} \neq 0$. Then the system (5.1) is exponentially stable in each state space among the Banach spaces $X_{r}, 1<r<\infty$, and $X_{\infty}=\left\{f \in(C[0,1])^{2} \mid f_{1}(0)=f_{2}(1)=0\right\}$ equipped with the uniform topology.

Proof of Proposition 5.1. Let $\theta=k\left(m_{2} m_{3}-m_{1} m_{4}\right)$ with $k>0$. It is easy to see that the matrix $\Lambda_{\theta}$ is positive for each $k>0$ such that

$$
k<\frac{1}{m_{1} m_{2}} .
$$

Therefore there exist a $\delta>0$ and a $\theta^{*} \in \mathbb{R} \backslash\{0\}$ with $\left|\theta^{*}\right|$ sufficiently small such that

$$
\dot{V}_{\theta^{*}}(\phi(t)) \leq-2 \delta V_{\theta^{*}}(\phi(t))
$$

or, equivalently

$$
V_{\theta^{*}}(\phi(t)) \leq \mathrm{e}^{-2 \delta t} V_{\theta^{*}}\left(\phi^{0}\right), \quad t \geq 0
$$

Using the fact that $\mathrm{e}^{-2\left|\theta^{*}\right|}\|\phi\|_{r}^{2} \leq V_{\theta^{*}}(\phi) \leq \mathrm{e}^{2\left|\theta^{*}\right|}\|\phi\|_{r}^{2}$, we get easily the exponential stability in each $X_{r}$, $1<r<\infty$,

$$
\left\|T_{r}(t) \phi^{0}\right\|_{r} \leq M_{\theta^{*}} \mathrm{e}^{-\delta t}\left\|\phi^{0}\right\|_{r} \quad \forall t \geq 0
$$

where the constant $M_{\theta^{*}} \geq 1$ is independent of $r$.

Now let the state space be $X_{\infty}=\left\{f \in(C[0,1])^{2} \mid f_{1}(0)=f_{2}(1)=0\right\}$ equipped with the uniform topology: $\|f\|_{X_{\infty}}=\max _{x \in[0,1]}\left\{\left|f_{1}(x)\right|,\left|f_{2}(x)\right|\right\}$. We define the unbounded operator $\widetilde{A}_{\infty}$ by

$$
D\left(\widetilde{A}_{\infty}\right)=\left\{f \in\left(C^{1}[0,1]\right)^{2} \cap X_{\infty} \mid A_{r} f+B_{r} f \in X_{\infty}\right\}
$$


and for each $f \in D\left(\widetilde{A}_{\infty}\right)$,

$$
\widetilde{A}_{\infty} f=A_{r} f+B_{r} f
$$

We prove that $\widetilde{A}_{\infty}$ is the generator of a $C^{0}$ semigroup on $X_{\infty}$ satisfying

$$
\left\|T_{\infty}(t) \phi^{0}\right\|_{\infty} \leq M_{\theta^{*}} \mathrm{e}^{-\delta t}\left\|\phi^{0}\right\|_{\infty}
$$

for the same constants $M_{\theta^{*}}$ and $\delta$ as in (5.13). Note that for any $f \in X_{\infty},\|f\|_{\infty}^{2}=\lim _{r \rightarrow \infty}\|f\|_{r}^{2}=\left\|f_{1}\right\|_{\infty}^{2}+$ $\left\|f_{2}\right\|_{\infty}^{2}$. As the norm $\|\cdot\|_{\infty}$ is equivalent to $\|\cdot\|_{X_{\infty}}$, the inequality (5.14) means the exponential stability of (5.1) in the state space $X_{\infty}$ with the uniform topology.

We have only to prove (5.14). For this purpose we use the fact that the function $r \rightarrow\|f\|_{r}$ is continuous from $(1, \infty]$ to $[0, \infty)$ for any fixed $f \in X_{\infty}$. It is easy to see that $\tilde{A}_{\infty}$ is the generator of a $C^{0}$ semigroup on $X_{\infty}$. Since $X_{\infty} \subset X_{r}$ and $D\left(A_{\infty}\right) \subset D\left(A_{r}\right)$ for $1<r<\infty$, taking limit in (5.13) as $r$ goes to $\infty$ we prove (5.14).

Remark 5.1. Even if $m_{1} m_{4}-m_{2} m_{3}=0$, the conclusion of the Proposition 5.1 is still true. It is proved by constructing another Lyapunov functional. Indeed let $r \in] 1, \infty\left[\right.$ and let $s=\frac{r}{r-1}$. Since $A_{r}+B_{r}$ is the generator of the semigroup $T_{r}(t)$ in the reflexive Banach space $X_{r}$, its dual $A_{r}^{*}+B_{r}$ is the generator of the dual semigroup $T_{r}^{*}(t)$ in the dual space $X_{r}^{*}=X_{s}$. Recall that the duality product of $X_{r} / X_{s}$ is given by

$$
\langle f, g\rangle_{X_{r}, X_{s}}=\int_{0}^{1}\left[f_{1}(x) g_{1}(x)+f_{2}(x) g_{2}(x)\right] \mathrm{d} x, \quad \forall f \in X_{r}, g \in X_{s} .
$$

The dual operator $A_{r}^{*}$ on $X_{s}$ is defined by

$$
A_{r}^{*} g=\left(\begin{array}{ll}
m_{1} & 0 \\
0 & -m_{2}
\end{array}\right) g^{\prime}, \quad D\left(A_{r}^{*}\right)=\left\{g \in\left(W^{1, s}(0,1)\right)^{2} \mid g_{1}(1)=g_{2}(0)=0\right\} .
$$

Let us consider $\phi^{0} \in D\left(A_{r}^{*}\right), \phi(t)=T_{r}^{*}(t) \phi^{0}$ is solution of the initial value problem:

$$
\left\{\begin{array}{l}
\dot{\phi}(t)=\left(A_{r}^{*}+B_{r}\right) \phi(t) \\
\phi(0)=\phi^{0} .
\end{array}\right.
$$

First apply the linear transformation to the evolution equation (5.16):

$$
\left\{\begin{array}{l}
\psi_{1}(t)=m_{3}^{\frac{1}{2 r}-\frac{1}{2 s}} \phi_{1} \\
\psi_{2}(t)=m_{4}^{\frac{1}{2 r}-\frac{1}{2 s}} \phi_{2} .
\end{array}\right.
$$

Then the PDE satisfied by $\left(\psi_{1}, \psi_{2}\right)$ takes the form:

$$
\left\{\begin{array}{l}
\frac{\partial}{\partial t}\left(\begin{array}{l}
\psi_{1} \\
\psi_{2}
\end{array}\right)=\left(\begin{array}{cc}
m_{1} & 0 \\
0 & -m_{2}
\end{array}\right) \frac{\partial}{\partial x}\left(\begin{array}{l}
\psi_{1} \\
\psi_{2}
\end{array}\right)+\left(\begin{array}{cc}
-m_{3} & m_{3}^{\frac{1}{r}} m_{4}^{\frac{1}{s}} \\
\frac{1}{s} m_{3}^{\frac{1}{r}} & -m_{4}
\end{array}\right)\left(\begin{array}{l}
\psi_{1} \\
\psi_{2}
\end{array}\right), \\
\psi_{1}(1, t)=0, \quad \psi_{2}(0, t)=0
\end{array}\right.
$$

Consider the Lyapunov functional

$$
W_{\theta}(\psi)=\int_{0}^{1}\left[p_{\theta}(x)\left|\psi_{1}(x, t)\right|^{s}+q_{\theta}(x)\left|\psi_{2}(x, t)\right|^{s}\right] \mathrm{d} x, \quad \theta>0
$$


with $p_{\theta}(x)=2-\mathrm{e}^{-\theta x}$ and $q_{\theta}(x)=2+\mathrm{e}^{-\theta x}$. By simple computations we find the time derivative of $W_{\theta}(\psi)$ along each trajectory of (5.18) as follows:

$$
\begin{aligned}
\dot{W}_{\theta}(\psi)= & -m_{1}\left|\psi_{1}(0, t)\right|^{s}-m_{1} \theta \int_{0}^{1} \mathrm{e}^{-\theta x}\left|\psi_{1}\right|^{s} \mathrm{~d} x-s m_{3} \int_{0}^{1} p_{\theta}(x)\left|\psi_{1}\right|^{s} \mathrm{~d} x \\
& +s m_{3}^{\frac{1}{r}} m_{4}^{\frac{1}{s}} \int_{0}^{1} p_{\theta}(x)\left|\psi_{1}\right|^{s-1} \operatorname{sign}\left(\psi_{1}\right) \psi_{2} \mathrm{~d} x \\
& -m_{2} q_{\theta}(1)\left|\psi_{2}(1, t)\right|^{s}-m_{2} \theta \int_{0}^{1} \mathrm{e}^{-\theta x}\left|\psi_{2}\right|^{s} \mathrm{~d} x-s m_{4} \int_{0}^{1} q_{\theta}(x)\left|\psi_{2}\right|^{s} \mathrm{~d} x \\
& +s m_{3}^{\frac{1}{s}} m_{4}^{\frac{1}{r}} \int_{0}^{1} q_{\theta}(x)\left|\psi_{2}\right|^{s-1} \operatorname{sign}\left(\psi_{2}\right) \psi_{1} \mathrm{~d} x .
\end{aligned}
$$

The following Young's inequality [16], p. 61, will be made use of in the upcoming estimates: for every $a>0$, $b>0, p>0$ and $q>0$ such that $\frac{1}{p}+\frac{1}{q}=1$,

$$
a b \leq \frac{a^{p}}{p}+\frac{a^{q}}{q}
$$

Let $p=s, q=r, a=m_{3}^{1 / s}\left[\int_{0}^{1}\left|\phi_{1}\right|^{s} \mathrm{~d} x\right]^{1 / s}$ and $b=m_{4}^{1 / r}\left[\int_{0}^{1}\left|\phi_{2}\right|^{s} \mathrm{~d} x\right]^{1 / r}$ and let us apply (5.21). We exchange $s$ and $r$ and apply again (5.21) to prove the following inequality

$$
\begin{gathered}
m_{3} \int_{0}^{1}\left|\phi_{1}\right|^{s} \mathrm{~d} x+m_{4} \int_{0}^{1}\left|\phi_{2}\right|^{s} \mathrm{~d} x=\frac{m_{3}}{s} \int_{0}^{1}\left|\phi_{1}\right|^{s} \mathrm{~d} x+\frac{m_{4}}{r} \int_{0}^{1}\left|\phi_{2}\right|^{s} \mathrm{~d} x+\frac{m_{3}}{r} \int_{0}^{1}\left|\phi_{1}\right|^{s} \mathrm{~d} x+\frac{m_{4}}{s} \int_{0}^{1}\left|\phi_{2}\right|^{s} \mathrm{~d} x \geq \\
m_{3}^{1 / s} m_{4}^{1 / r}\left[\int_{0}^{1}\left|\phi_{1}\right|^{s} \mathrm{~d} x\right]^{1 / s}\left[\int_{0}^{1}\left|\phi_{2}\right|^{s} \mathrm{~d} x\right]^{1 / r}+m_{3}^{1 / r} m_{4}^{1 / s}\left[\int_{0}^{1}\left|\phi_{1}\right|^{s} \mathrm{~d} x\right]^{1 / r}\left[\int_{0}^{1}\left|\phi_{2}\right|^{s} \mathrm{~d} x\right]^{1 / s} \cdot
\end{gathered}
$$

By $s=\frac{r}{r-1}$ and the classical Hölder inequality we have

$$
\int_{0}^{1}\left|\phi_{1}\right|^{s-1}\left|\phi_{2}\right| \mathrm{d} x \leq\left[\int_{0}^{1}\left|\phi_{2}\right|^{s} \mathrm{~d} x\right]^{1 / s}\left[\int_{0}^{1}\left|\phi_{1}\right|^{s} \mathrm{~d} x\right]^{1 / r} .
$$

Using the inequalities (5.22) and (5.23) in (5.20), we obtain the estimate:

$$
\dot{W}_{\theta}(\psi) \leq-\left(m_{1} \theta-2 m_{3} s\right) \int_{0}^{1} \mathrm{e}^{-\theta x}\left|\psi_{1}\right|^{s} \mathrm{~d} x-m_{2} \theta \int_{0}^{1} \mathrm{e}^{-\theta x}\left|\psi_{2}\right|^{s} \mathrm{~d} x .
$$

Therefore, choosing $\theta=\theta^{*}=1 / m_{2}+\left(1+2 m_{3} s\right) / m_{1}$ we get

$$
\dot{W}_{\theta^{*}}(\psi) \leq-\int_{0}^{1} \mathrm{e}^{-\theta^{*} x}\left|\psi_{1}\right|^{s} \mathrm{~d} x-\int_{0}^{1} \mathrm{e}^{-\theta^{*} x}\left|\psi_{2}\right|^{s} \mathrm{~d} x .
$$

By simple majorations and by (5.19) we obtain

$$
\dot{W}_{\theta^{*}}(\psi) \leq-\frac{1}{3} \mathrm{e}^{-\theta^{*}} W_{\theta^{*}}(\psi),
$$

which implies, by integration, the estimate

$$
W_{\theta^{*}}(\psi) \leq \mathrm{e}^{-\lambda t} W_{\theta^{*}}(\psi(0))
$$


for $\lambda=\frac{1}{3} \mathrm{e}^{-\theta^{*}}>0$. Since $\left\|T_{r}(t)\right\|_{\mathcal{L}\left(X_{r}\right)}=\left\|T_{r}^{*}(t)\right\|_{\mathcal{L}\left(X_{s}\right)}$, the exponential stability is proved in each $X_{r}$, $1<r<\infty$ :

$$
\left\|T_{r}(t) \phi_{0}\right\|_{r} \leq M \mathrm{e}^{-\lambda(1-1 / r) t}\left\|\phi_{0}\right\|_{r} \quad \forall t \geq 0, \phi_{0} \in D\left(A_{r}\right),
$$

where the constant $M \geq 1$ is independent of $r$ and the exponent has a non-zero finite limit when $r$ goes to $\infty$.

Remark 5.2. The above method can be generalized to prove the exponential stability in $X_{r}, 1<r \leq \infty$, for systems of type (2.1) where the matrices $a(x)$ and $b(x)$ are constants with $b(x)$ symmetric, $D_{0}=0$, and $D_{1}=0$.

\subsection{Numerical simulations}

Let us consider the contra-flow heat exchanger system described by the following PDE:

$$
\begin{aligned}
& \frac{\partial}{\partial t}\left(\begin{array}{l}
R_{1} \\
R_{2}
\end{array}\right)=\left(\begin{array}{cc}
-m_{1} & 0 \\
0 & m_{2}
\end{array}\right) \frac{\partial}{\partial x}\left(\begin{array}{l}
R_{1} \\
R_{2}
\end{array}\right)+\left(\begin{array}{cc}
-m_{3} & m_{3} \\
m_{4} & -m_{4}
\end{array}\right)\left(\begin{array}{l}
R_{1} \\
R_{2}
\end{array}\right) \\
& R_{1}(0, t)=0, \quad R_{2}(1, t)=0 \\
& R_{1}(x, 0)=\phi_{1}(x), \quad R_{2}(x, 0)=\phi_{2}(x) .
\end{aligned}
$$

From the theoretical point of view, $\left(R_{1}(x, t), R_{2}(x, t)\right)$ tends exponentially to zero in the uniform norm as $t \rightarrow \infty$. Moreover we can check it by simulation. Indeed we take $m_{1}=m_{2}=m_{3}=1, m_{4}=4, T_{10}=20$ and $T_{20}=80$, and the initial condition as follows

$$
\begin{gathered}
\phi_{1}(x)=T_{10}\left(k_{1} x^{2}+1\right) \mathrm{e}^{-x^{2}}-\frac{\left(4 \mathrm{e}^{3}-\mathrm{e}^{3 x}\right) T_{10}+\left(\mathrm{e}^{3 x}-1\right) T_{20}}{4 \mathrm{e}^{3}-1} \\
\phi_{2}(x)=T_{20} \mathrm{e}^{-\frac{(1-x)^{2}}{t_{1}}}-\frac{4\left(\mathrm{e}^{3}-\mathrm{e}^{3 x}\right) T_{10}+\left(4 \mathrm{e}^{3 x}-1\right) T_{20}}{4 \mathrm{e}^{3}-1}
\end{gathered}
$$

where $k_{1}=\frac{T_{20}}{T_{10}} \mathrm{e}^{1}-1$ and $t_{1}=\frac{-1}{\log \frac{T_{10}}{T_{20}}}$. The initial condition is chosen to satisfy the compatibility condition. The system (5.26) is solved by the finite difference method applied on the derivative with respect to $x$. The partial differential equation is approximated by ordinary differential equations (ODE) with respect to the time $t$. The ODE system is solved by Matlab R12. Figures 1 and 2 represent the evolution of the profiles of the temperatures $R_{1}$ and $R_{2}$ along the exchanger. We observe that the system under consideration is stable, since the solution of (5.26) converges to zero in some finite time, which is in conformity with the theoretical result. Figure 3 shows the dynamical evolution of the uniform norm of the state variables $R(x, t)$. The system is apparently dissipative with the respect to the uniform norm and its solution converges monotonically to zero in the uniform norm.

\section{Conclusions}

In this paper we have presented a brief and concise review on the applications of Lyapunov's direct method and its development in the studies of asymptotic behaviour of dynamical systems, in particular, of distributed parameter systems governed by hyperbolic PDEs. To meet the growing demand from chemical engineering we have investigated a specific class of symmetric hyperbolic systems in terms of stability. We have presented a new proof of some stability result in [31] by constructing Lyapunov functionals. The presented proof is a generalization of a previous one [40] in the sense that no symmetry condition is asked for the matrix $b(x)$. Compared with the method of characteristics used in [31] the Lyapunov method is direct, simple and admits extension to cases of nonlinearities, multiple spatial variables and more general Banach spaces. To show potential applications of the proved theorem we have worked out a heat transfer process - counter current heat exchangers. Based on the example we have shown how nonlinearities can be taken into account easily by the constructed Lyapunov functionals. We have also applied the Lyapunov's method to prove exponential stability of some 


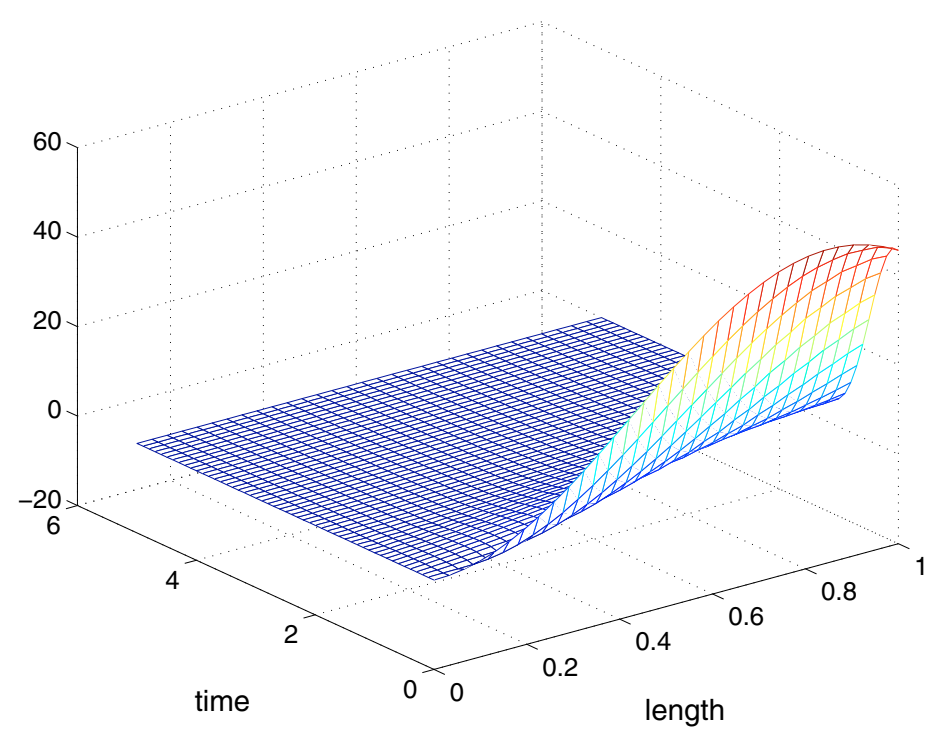

Figure 1. Dynamical evolution of $R_{1}(x, t)$.

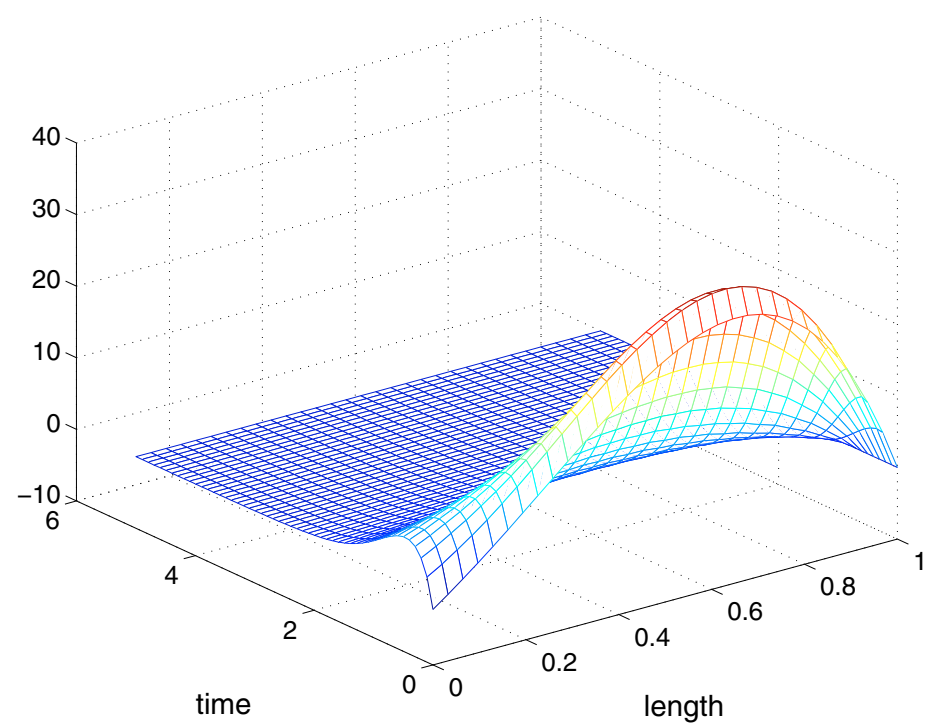

Figure 2. Dynamical evolution of $R_{2}(x, t)$.

hyperbolic PDEs with two space variables which are related to the classical D'Alembert equation. Our stability results have been compared and contrasted with existing results in the literature.

Through examples we have endeavoured to give an insight into effective applications of the Lyapunov method in the framework of Banach spaces: $L^{p}$ spaces and the space of continuous functions. The Lyapunov functionals that we have elaborated would be potentially utilizable for investigating stability of nonlinear systems in a more general framework of operators ( $c f$. [4]) as well as for doing robust feedback control [8,9,34]. 


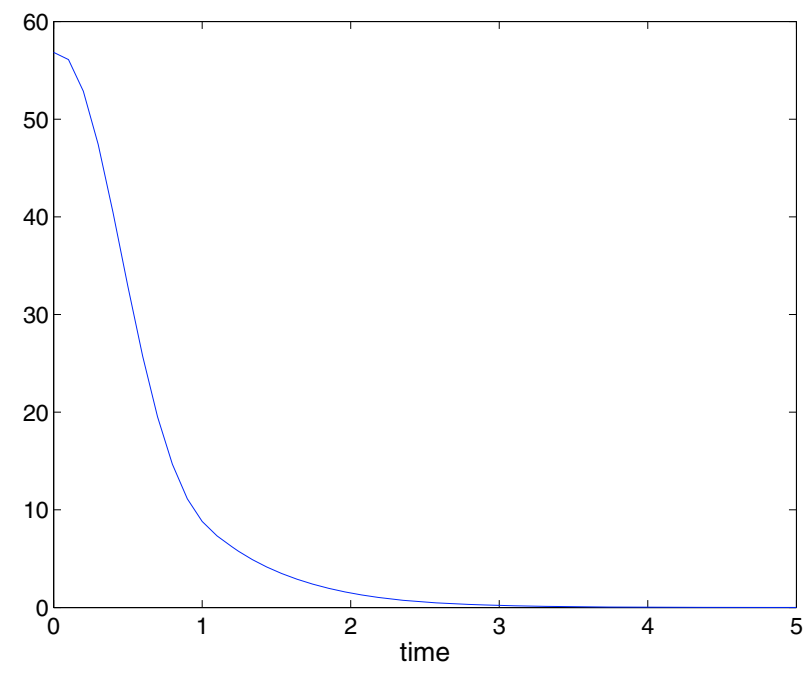

Figure 3. Dynamical evolution of $\left(\left\|R_{1}(., t)\right\|_{\infty}^{2}+\left\|R_{2}(., t)\right\|_{\infty}^{2}\right)^{1 / 2}$.

However, in the framework of operators the stability problem is more complex even for the class of symmetric hyperbolic systems (2.1). In that framework the asymptotic decay of solutions will be exponential or polynomial according to the case. The present setting is far from proposing a necessary and sufficient condition of asymptotic stability for the studied systems. In this aspect more sophisticated notions based on the micro-local behaviour analysis would be necessary to be made use of for a complete theory of stability $(c f .[6,33]$ and the related references there).

Acknowledgements. We would like to thank Professor Claude Lobry (University of Nice) for numerous discussions on the subject of the paper. We thank also the reviewers for their constructive comments on an earlier version and the interesting references that they have suggested to us. Our research program has been started in 1999 with a funding support of the CIMPA (Centre International de Mathématiques Pures et Appliquées) and accomplished in 2007 during the visit of Dr. A. Tchousso in France with a postdoctoral fellowship from the AUF (Agence Universitaire de la Francophonie).

\section{REFERENCES}

[1] F. Alabau, Stabilisation frontière indirecte de systèmes faiblement couplés. C.R. Acad. Sci. Paris Série I 328 (1999) $1015-1020$.

[2] F. Alabau, P. Cannarsa and V. Komornik, Indirect internal stabilization of weakly coupled evolution equations. J. Evol. Eq. 2 (2002) $127-150$.

[3] J. Baillieul and M. Levi, Rotational elastic dynamics. Physica D 27 (1987) 43-62.

[4] S. Bianchini, B. Hanouzet and R. Natalini, Asymptotic behaviour of smooth solutions for partially dissipative hyperbolic systems with a convex entropy. Comm. Pure Appl. Math. 60 (2007) 1559-1622.

[5] H. Brezis, Analyse fonctionnelle : théorie et applications. Masson, Paris (1983).

[6] N. Burq and G. Lebeau, Mesure de défaut de compacité, application au système de Lamé. Ann. Sci. École Norm. Sup. 34 (2001) 817-870.

[7] A. Chapelon and C.Z. Xu, Boundary control of a class of hyperbolic systems. Eur. J. Control 9 (2003) 589-604.

[8] J.M. Coron, B. d'Andréa-Novel and G. Bastin, A Lyapunov approach to control irrigation canals modeled by Saint-Venant equations. European Control Conference ECC'99, Karlsruhe, September (1999).

[9] J.M. Coron, B. d'Andréa-Novel and G. Bastin, A strict Lyapunov function for boundary control of hyperbolic systems of conservation laws. IEEE Trans. Automat. Control 52 (2007) 2-11.

[10] R.F. Curtain and H.J. Zwart, An introduction to infinite-dimensional linear systems theory. Springer-Verlag, New York (1995).

[11] B. d'Andréa-Novel, Commande non linéaire des robots. Hermès (1988).

[12] P. Freitas, Stability results for the wave equation with indefinite damping. J. Diff. Eq. 132 (1996) 338-353. 
[13] J.M. Greenberg and T.T. Li, The effect of boundary damping for the quasilinear wave equation. J. Diff. Eq. 52 (1984) 66-75.

[14] C.D. Immanuel, C.F. Cordeiro, S.S. Sundaram, E.S. Meadows, T.J. Crowley and F.J. Doyle III, Modeling of particule size distribution in emulsion co-polymerization: comparaison with experimental data and parameter sensitivity studies. Comput. Chem. Eng. 26 (2002) 1133-1152.

[15] B. Kalitine, Sur la stabilité des ensembles compacts positivement invariants des systèmes dynamiques. RAIRO-Automatique 16 (1982) $275-286$.

[16] L.V. Kantorovich and G.P. Akilov, Functional analysis in normed spaces. Pergamon Press, Oxford (1964).

[17] V. Komornik, Exact controllability and stabilization: the multiplier method, Research in Applied Mathematics. Series Editors: P.G. Ciarlet and J.L. Lions, Masson, Paris (1994).

[18] V. Komornik and E. Zuazua, A direct method for the boundary stabilization of the wave equation. J. Math. Pures Appl. 69 (1990) 33-54.

[19] J.P. LaSalle and S. Lefschetz, Stability by Liapunov's direct method with applications. Academic Press, New York (1961).

[20] P.D. Lax and R.S. Phillips, Local boundary conditions for dissipative symmetric linear differential operators. Comm. Pure Appl. Math. 13 (1960) 427-455.

[21] T.-T. Li, Global classical solutions for quasilinear hyperbolic systems, Research in Applied Mathematics. John Wiley \& Sons, New York (1994).

[22] A. Liapunov, Problème général de la stabilité du mouvement. Princeton University Press, Princeton, New Jersey (1947).

[23] J. Liéto, Génie chimique à l'usage des chimistes. Lavoisier, Paris (1998).

[24] Z.H. Luo, B.Z. Guo and O. Morgul, Stability and stabilization of infinite dimensional systems with applications. Springer, London (1999).

[25] Nasa Technical Memorandum, Progress Report No. 8, in Proceedings of the twenty-fourth seminar on space flight and guidance theory, NASA George G. Marshall space flight center, Huntsville, Alabama, June 3 (1966).

[26] R. Outbib and G. Sallet, Stabilizability of the angular velocity of a rigid body revisited. Systems Control Lett. 18 (1992) 93-98.

[27] A. Pazy, Semigroups of Linear Operators and Applications to Partial Differential Equations. Springer-Verlag, New York (1983).

[28] F. Puel, G. Févotte and J.P. Klein, Simulation and analysis of industrial cristallization processes through multidimensional population balance equation. Part 1: A resolution algorithm based on the method of classes. Chem. Engrg. Sci. 58 (2003) $3715-3727$.

[29] D. Ramkrishna and A.W. Mahoney, Population balancemodeling. Promise for the future. Chem. Engrg. Sci. 57 (2002) 595-606.

[30] B. Rao, Le taux optimal de décroissance de l'énergie dans l'équation de poutre de Rayleigh. C. R. Acad. Sci. Paris 325 (1997) $737-742$.

[31] J. Rauch and M. Taylor, Exponential decay of solutions to hyperbolic equations in bounded domain. Indiana Univ. Math. J. 24 (1974) 79-86.

[32] D.L. Russell, Controllability and stabilizability theory for linear partial differential equations: recent progress and open questions. SIAM Rev. 20 (1978) 639-739.

[33] D. Serre, Solvability of hyperbolic IBVPS through filtering. Methods Appl. Anal. 12 (2005) 253-266.

[34] E. Sontag and H. Sussmann, Further comments on the stabilizability of the angular velocity of a rigid body. Systems Control Lett. 12 (1988) 213-217.

[35] G. Szegö, On the application of Zubov's method of constructing Liapunov functions for nonlinear control systems. Transaction of ASME Journal of Basic Eng. Series D 85 (1963) 137-142.

[36] A. Tchousso, Étude de la stabilité asymptotique de quelques modèles de transfert de chaleur. Ph.D. thesis, University of Claude Bernard - Lyon 1, France (2004).

[37] A. Tchousso and C.Z. Xu, Exponential stability of symmetric hyperbolic systems using Lyapunov functionals, in Proceedings of the 10th IEEE International Conference on Methods and Models in Automation and Robotics, Miedzyzdroje, Poland (2004) $361-364$.

[38] A.J. van der Schaft, Stabilization of Hamiltonian systems. Nonlinear Anal. Methods Appl. 10 (1986) 1021-1035.

[39] C.Z. Xu and G. Sallet, Exponential stability and transfer functions of a heat exchanger network. Rapport de Recherche de l'INRIA 3823 (1999) 1-21.

[40] C.Z. Xu and G. Sallet, Exponential stability and transfer functions of processes governed by symmetric hyperbolic systems. ESAIM: COCV 7 (2002) 421-442.

[41] C.Z. Xu, J.P. Gauthier and I. Kupka, Exponential stability of the heat exchanger equation, in Proceedings of the European Control Conference, Groningen, The Netherlands (1993) 303-307.

[42] E. Zuazua, Exact controllability for semilinear wave equations in one space dimension. Ann. Inst. H. Poincaré Anal. Non Linéaire 10 (1993) 109-129. 